\title{
Continuum in MDGC Technology: From Classical Multidimensional to Comprehensive Two-Dimensional Gas Chromatography
}

\section{Supporting Information}

Chadin Kulsing,${ }^{\dagger}$ Yada Nolvachai,${ }^{\dagger}$ Paul Rawson, ${ }^{\ddagger}$ David J. Evans,${ }^{\ddagger}$ and Philip J. Marriott ${ }^{\dagger *}$ ${ }^{\dagger}$ Australian Centre for Research on Separation Science, School of Chemistry, Monash University, Wellington Road, Clayton, VIC 3800, Australia.

Defence Science and Technology Group, 506 Lorimer Street, Fishermans Bend 3207, Australia

\section{Analytical Chemistry}




\section{Table of Content}

1. An illustration for the benefit of increasing $N_{i n j}$ in MDGC with previously reported experimental

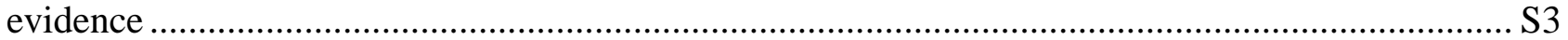

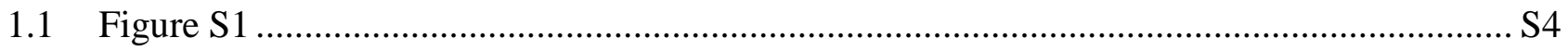

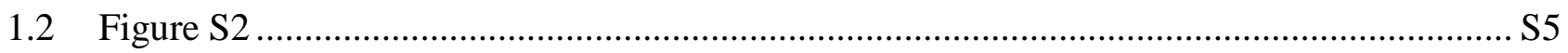

2. Additional Figures

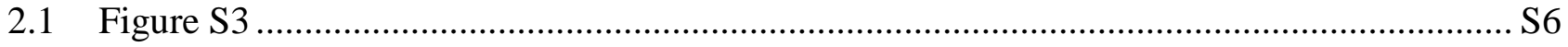

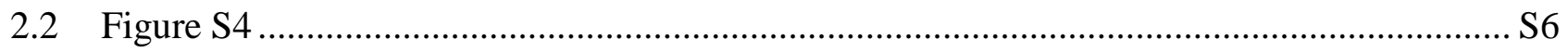

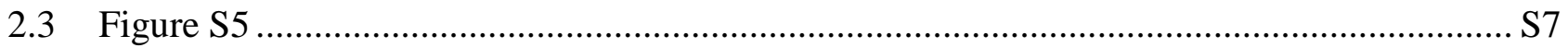

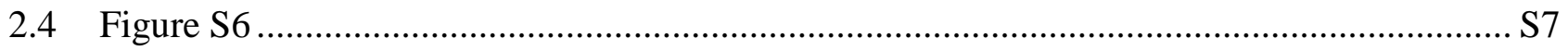

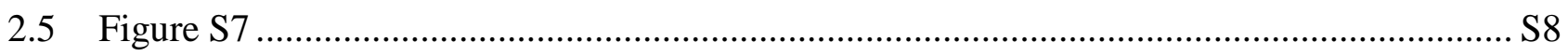

3. Additional Table

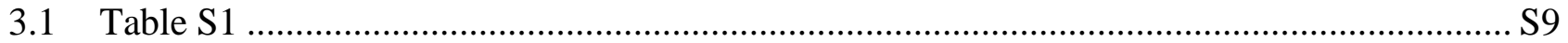

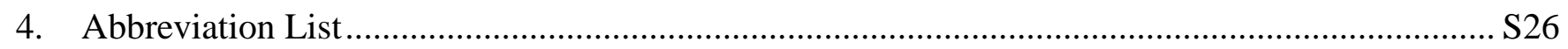

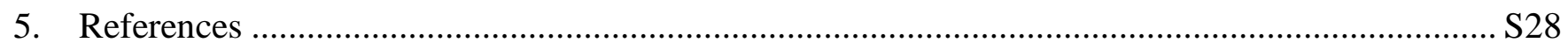




\section{An illustration for the benefit of increasing $N_{\mathrm{inj}}$ in MDGC with previously reported experimental evidence}

Conventional GC $\times \mathrm{GC}$ analysis requires $P_{M}$ to be at least equal to the minimum time required to complete ${ }^{2} \mathrm{D}$ separation without presence of wraparound $\left({ }^{2} t_{\mathrm{R}, \max }\right)$ where peaks (or part of the peaks) in one sampling coeluting during the next sampling in ${ }^{2} \mathrm{D}$ separation. Consequently, ${ }^{2} t_{\mathrm{R} \text {, max }}$ should be $<P_{\mathrm{M}}$. Practically, fast elution conditions are applied, e.g. by using a short ${ }^{2} \mathrm{D}$ column with $P_{\mathrm{M}}<6 \mathrm{~s}$. Whilst allowing high ${ }^{1} \mathrm{D}$ separation efficiency, such conditions invariably suppress ${ }^{2} \mathrm{D}$ separation efficiency. This constrains the difference in retention time of components that might be reliably resolved. Improved ${ }^{2} \mathrm{D}$ separation efficiency may be obtained by employing a long ${ }^{2} \mathrm{D}$ column which results in increasing ${ }^{2} t_{\mathrm{R} \text {,max }}$ (and thus permitting longer $P_{\mathrm{M}}$ ). However, separation with $P_{\mathrm{M}}$ exceeding peak width in ${ }^{1} \mathrm{D}$, e.g. $P_{\mathrm{M}}>0.5$ min, reduces ${ }^{1} \mathrm{D}$ efficiency due to peak broadening in ${ }^{1} \mathrm{D}$ separation caused by excessive sampling of peaks.

With the aim of reducing analysis time in MDGC with multiple sampling, as well as maintaining high resolution ${ }^{1} \mathrm{D}$ and ${ }^{2} \mathrm{D}$ separation, multiple $\mathrm{H} / \mathrm{C}$ with short sampling time $\left(t_{\text {samp }}\right)$ and rapid cycle time $\left(t_{c y c}\right)$ was performed by using DS prior to modulator operation. This technique performs multiple H/C processes contiguously arranged based on repetitive $t_{c y c}$, which corresponds to the ${ }^{2} t_{\mathrm{R} \text {, max }}, e . g$. every $1 \mathrm{~min}$. In this case $t_{\text {samp }}$ is practically set to be less than (or equal to) and proportional to the $t_{\mathrm{cyc}}, e . g . t_{\mathrm{samp}}=0.25,0.5$ or $1 \mathrm{~min}$ and $t_{c y c}=1 \mathrm{~min}$. Note that conventional GC $\times \mathrm{GC}$ employs $t_{s a m p}=t_{c y c}$. When $t_{c y c}>t_{s a m p}$, it is not possible to sample contiguous $\mathrm{H} / \mathrm{C}$ throughout the single run. In this case, coverage of the whole sample requires additional injections. The number of injections $\left(N_{i n j}\right)$ is then equal to the ratio of $t_{c y c}$ to $t_{s a m p}$. Combination of one modulator and one DS (or 2 DS where one functioning as a modulator) in comprehensive MDGC enabled increasing $N_{i n j}$ which enables the application of shorter $t_{\text {samp }}$ and at the same time applying a long ${ }^{2} \mathrm{D}$ column (improved ${ }^{2} \mathrm{D}$ resolution). Consequently, ${ }^{1} \mathrm{D}$ resolution is maintained and fewer sampled components to be separated during ${ }^{2} \mathrm{D}$ separation as illustrated in Figure 2 (in the manuscript) for reduction of number of coeluting analytes on the ${ }^{2} \mathrm{D}$ separation at higher $N_{i n j}$.

An example illustrating the benefit of increasing $N_{i n j}$ with a fixed ${ }^{2} \mathrm{D}$ column length $\left({ }^{2} L\right)$ in MDGC is provided below for separation of 39 analytes where conventional peak capacity $\left(n_{c}={ }^{1} n_{c} \times{ }^{2} n_{c}\right)$ in separation can be enhanced. Providing that the $\mathrm{H} / \mathrm{C}$ time window is significantly larger than the analyte peak width, $n_{\mathrm{c}}$ linearly increases with $N_{i n j}$, as calculated from the number of grids shown in Figure S1. In this figure, $n_{c},{ }^{1} n_{c}$ and ${ }^{2} n_{c}$ 
are represented by the total number of bins, the total number of columns and the total number of rows in the grid-scale normalized 2D results, respectively. By counting the number of bins containing analyte(s) for each grid-scale normalized 2D result, the corresponding $P_{a p p}$ can be obtained. $P_{a p p}$ increases with the increasing $N_{i n j}$ being 24, 34 and 39 analytes from left to right, respectively.

\section{Improved separation performance in comprehensive}

\section{MDGC by increasing injection number $\left(N_{\text {inj }}\right)$ using Deans switch}

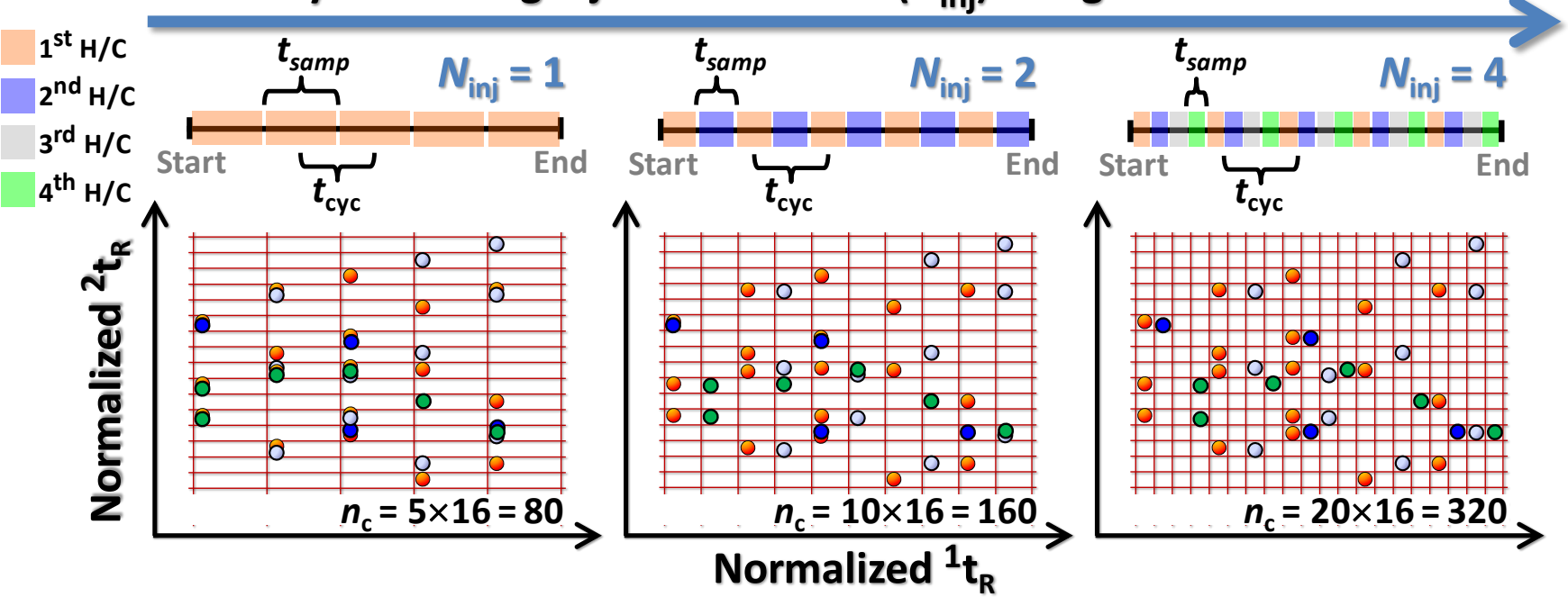

Figure S1. Example grid-scale normalized 2D results for separation of 39 analytes (represented by spheres with different colours) in MDGC using different $N_{i n j}=1,2$ and 4 (from left to right), corresponding to $t_{s a m p}$ $=t_{c y c}, 2 t_{\text {samp }}=t_{\mathrm{cyc}}$ and $4 t_{\text {samp }}=t_{\mathrm{cyc}}$, respectively. For $N_{i n j}=4$; the analyte to be separated in $\mathrm{H} / \mathrm{C} 1-4$ are coloured in orange, blue, grey and green, respectively. Note that $\mathrm{GC} \times \mathrm{GC}$ is the case when $N_{i n j}=1$.

This approach was applied for improved separation of thermally oxidized fuel sample, ${ }^{1,2}$ e.g. where the separation power from 1DGC appears (Figure S2A) to be insufficient for analysis of oxygenated compounds whilst these compounds were well resolved in multiple $\mathrm{H} / \mathrm{C}$ analysis with $N_{i n j}=10$ (Figure $\mathrm{S} 2 \mathrm{~B}$ ). 

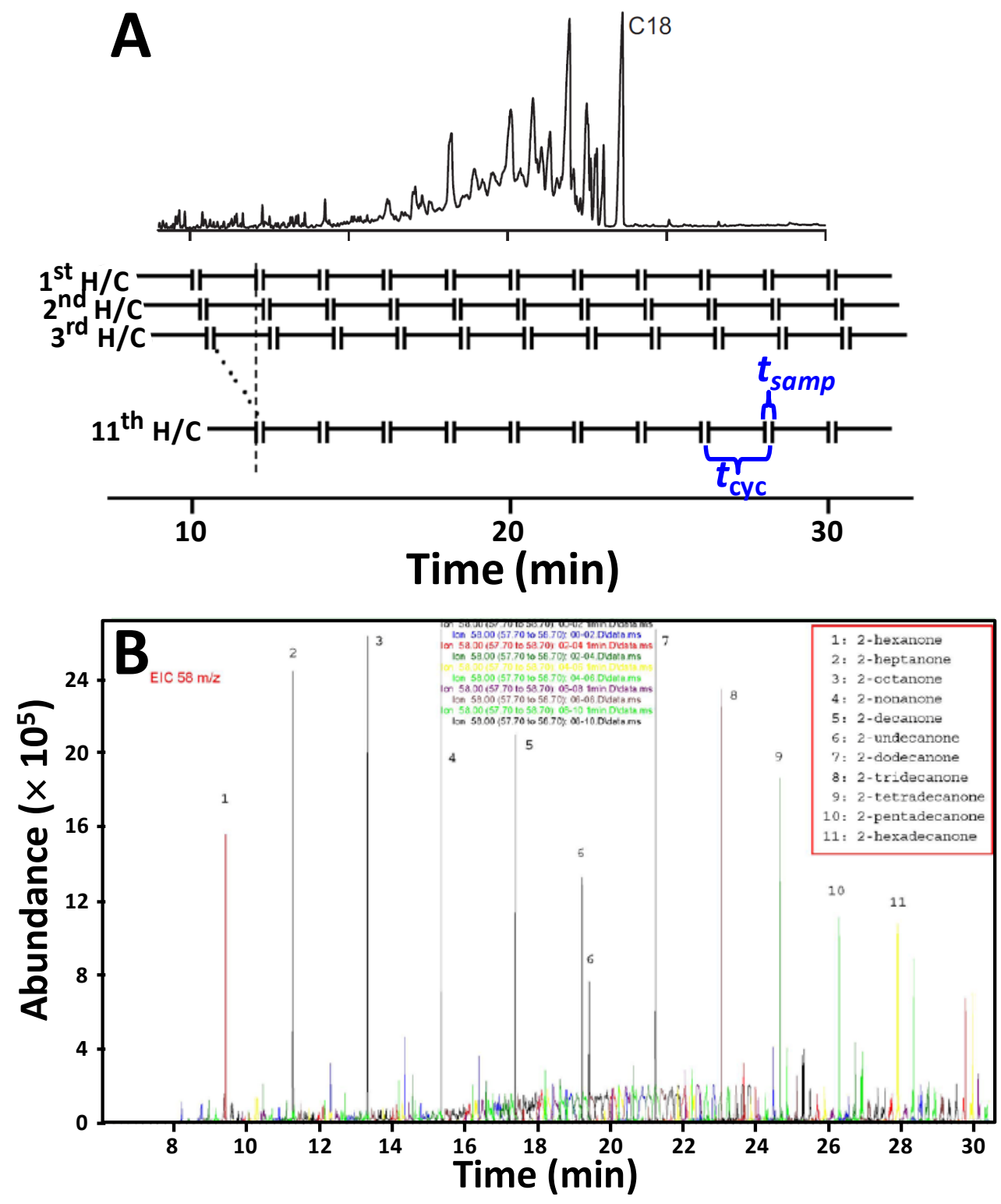

Figure S2. (A) 1DGC-MS total ion chromatogram of an algae-derived fuel oil with the latest major component is tentatively identified as $n$-octadecane and (B) overlaid 10 multiple $\mathrm{H} / \mathrm{C}$ MDGC results (extracted ion chromatograms) obtained with short sampling time $\left(t_{s a m p}\right)$ and rapid cycle time $\left(t_{c y c}\right)$ by using $t_{\text {samp }}=0.2 \mathrm{~min}$ and $t_{c y c}=2 \mathrm{~min}$, which requires 10 injections $\left(N_{i n j}=10\right)$ to complete the analysis covering the whole region of interest (e.g. from 10-32 min). Thus, the $1^{\text {st }}$ and $11^{\text {th }} \mathrm{H} / \mathrm{C}$ will sample the same region of the ${ }^{1} \mathrm{D}$ column separation. The figure was remade from the supplementary information reported in Mitrevski et al. ${ }^{1}$ 
2. Additional Figures
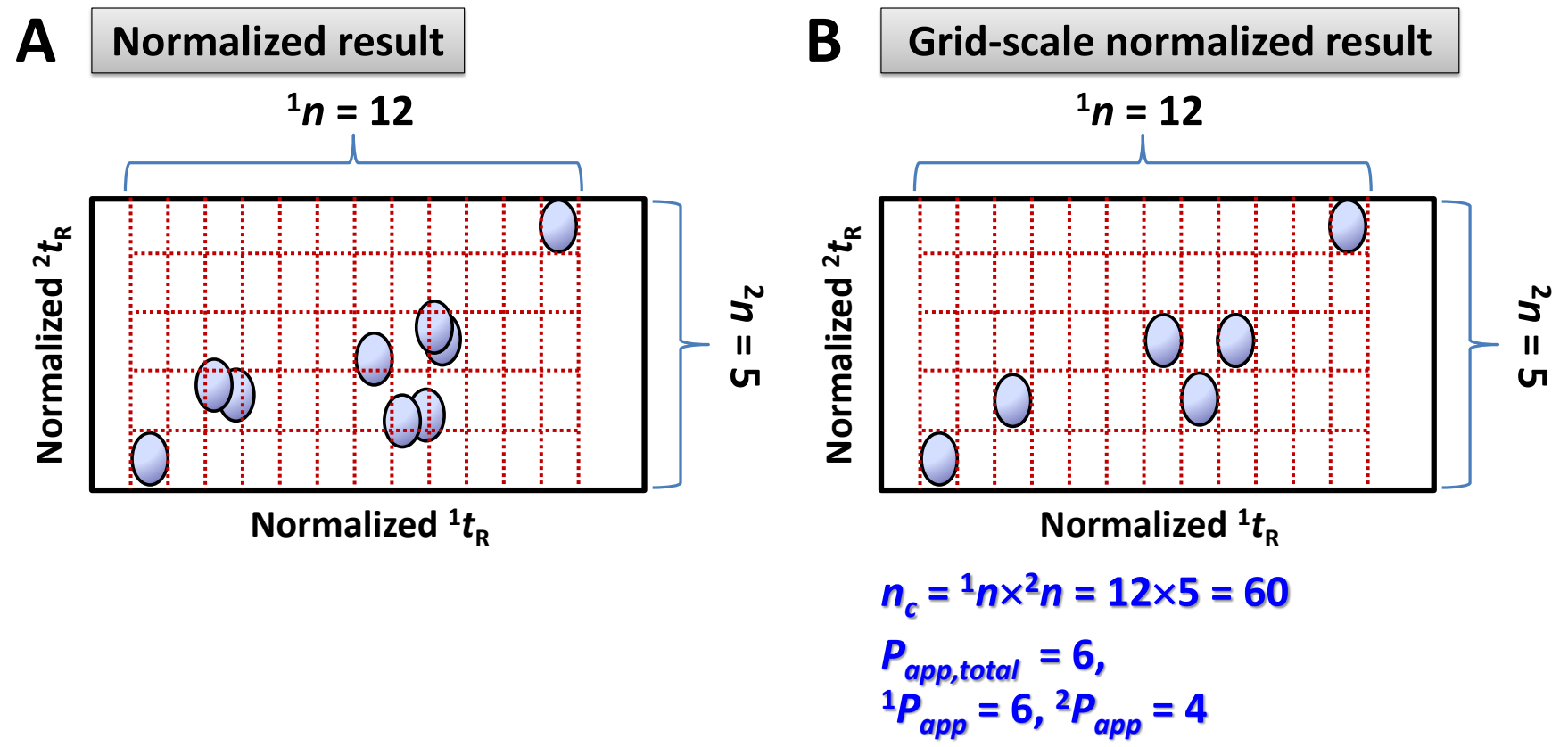

Figure S3. A diagram illustrating the difference between (A) "normalized" and (B) "grid-scale normalized" $2 \mathrm{D}$ results.

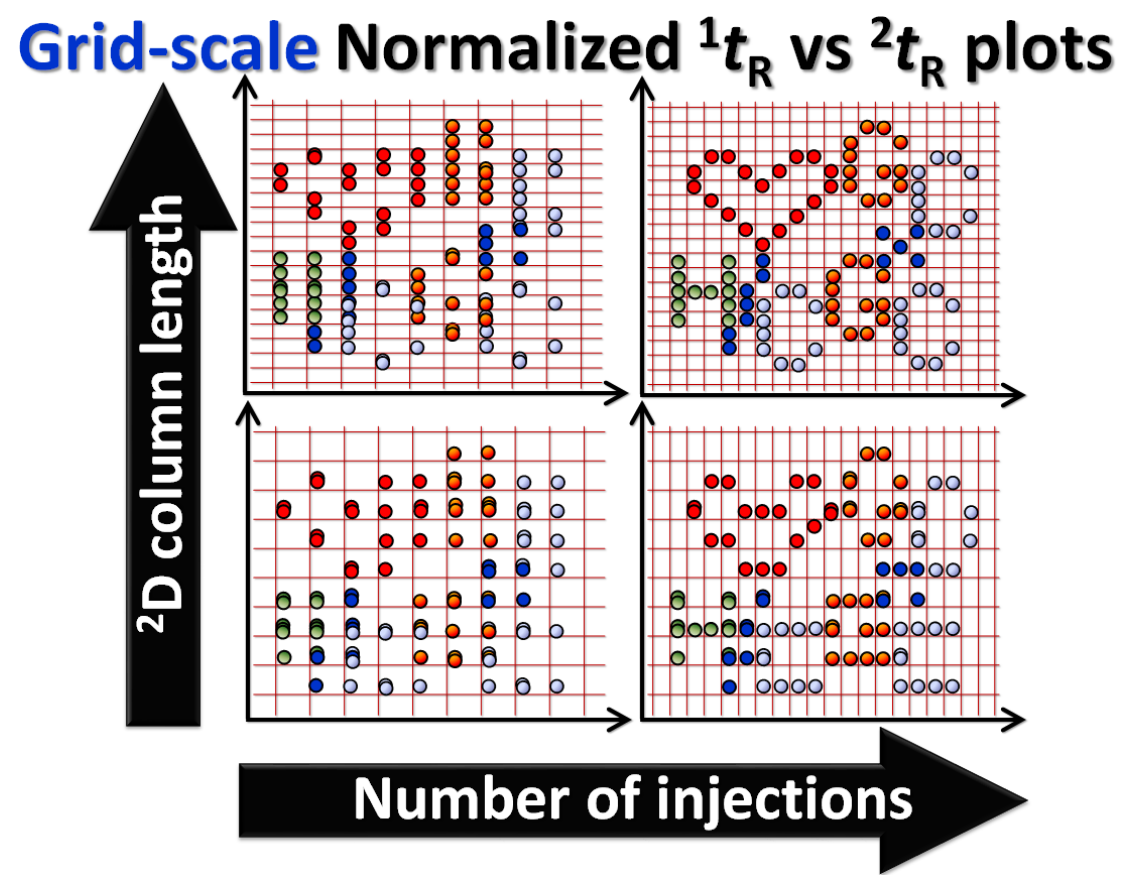

Figure S4. A diagram illustrating improved ${ }^{1} n_{c}$ (total numbers of columns of bins in grid-scale normalized $2 \mathrm{D}$ results), ${ }^{2} n_{c}$ (total numbers of rows of bins), $n_{c}$ (total numbers of bins) and $P_{\text {app,total }}$ (total numbers of bins containing analytes) by increasing $N_{i n j}$ and ${ }^{2} L$ which independently increase ${ }^{1} n_{c}$ and ${ }^{2} n_{c}$, respectively. Analytes are represented by spheres. 

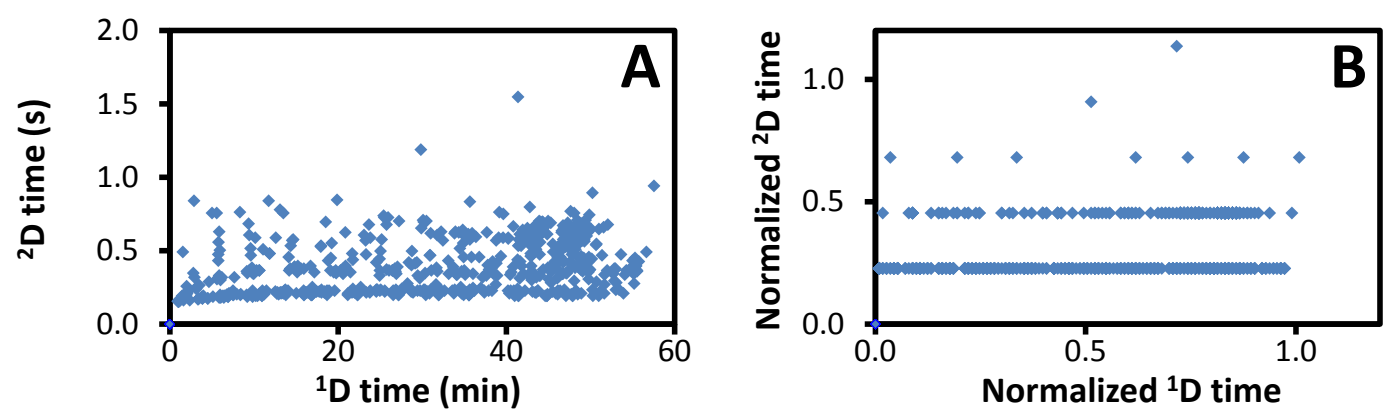
$5 \mathrm{~ms}(30 \mathrm{~m})$ WAX $(0.5 \mathrm{~m})$
$n=1$
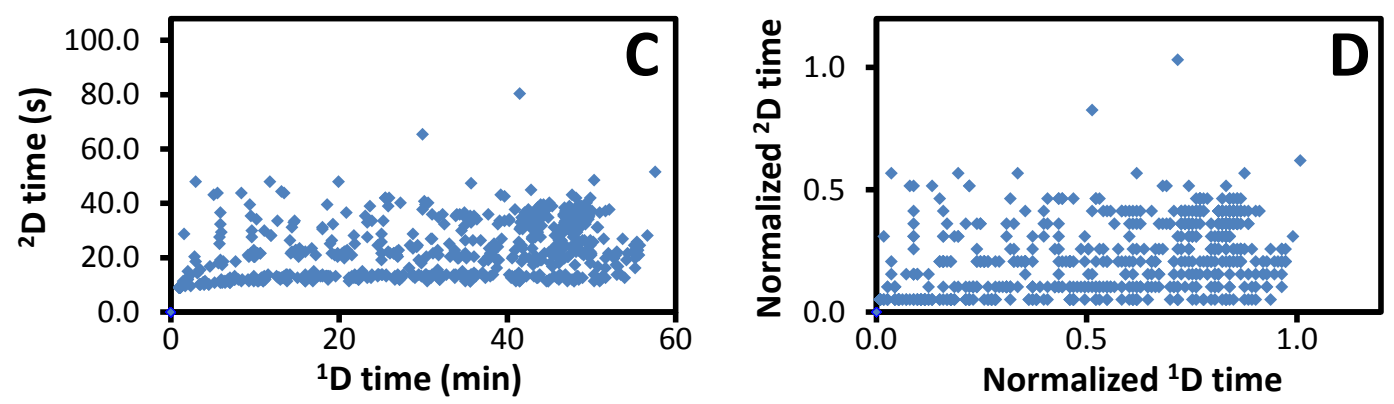

$5 \mathrm{~ms}(30 \mathrm{~m})$ WAX (30m)

$n=12$

Figure S5. Simulated ${ }^{1} t_{\mathrm{R}}$ vs ${ }^{2} t_{\mathrm{R}}$ plots for 681 analytes in kerosene separated on (A): ${ }^{1} \mathrm{D} 5 \mathrm{~ms}(30 \mathrm{~m})$ and ${ }^{2} \mathrm{D}$ WAX $(0.5 \mathrm{~m})$ with $n=1$ and (C): ${ }^{1} \mathrm{D} 5 \mathrm{~ms}(30 \mathrm{~m})$ and ${ }^{2} \mathrm{D}$ WAX $(30 \mathrm{~m})$ with $n=12$ with the corresponding grid-scale normalized chromatograms shown in (B) and (D), respectively.
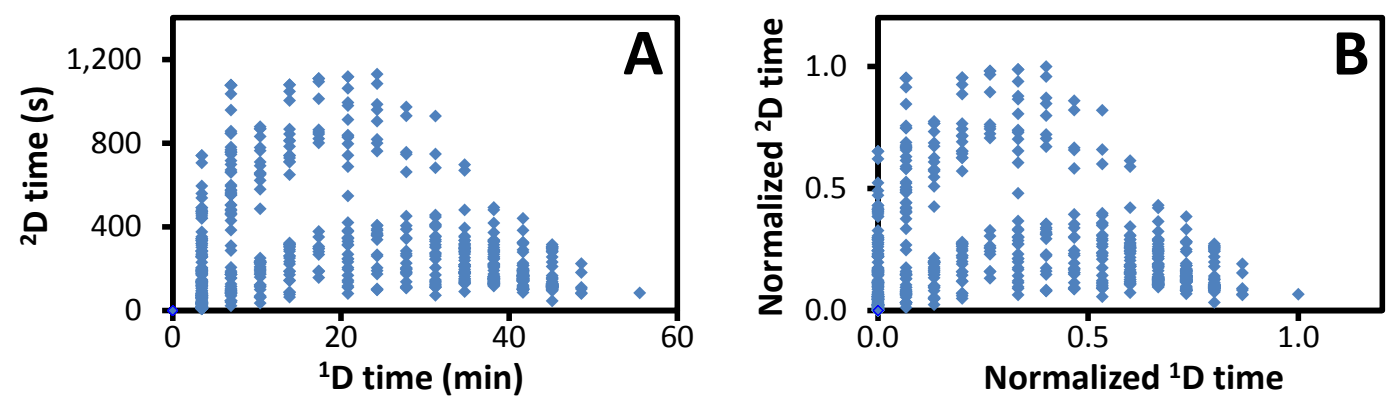

IL82 (30m) $5 \mathrm{~ms}(30 \mathrm{~m})$

$n=6$

Figure S6 Simulated ${ }^{1} t_{\mathrm{R}}$ vs ${ }^{2} t_{\mathrm{R}}$ plot for 681 analytes in kerosene separated on (A): ${ }^{1}$ D SLB-IL82 (30 m) and ${ }^{2} \mathrm{D} 5 \mathrm{~ms}(30 \mathrm{~m}$ ) with $n=6$ with the corresponding grid-scale normalized chromatogram shown in (B). 

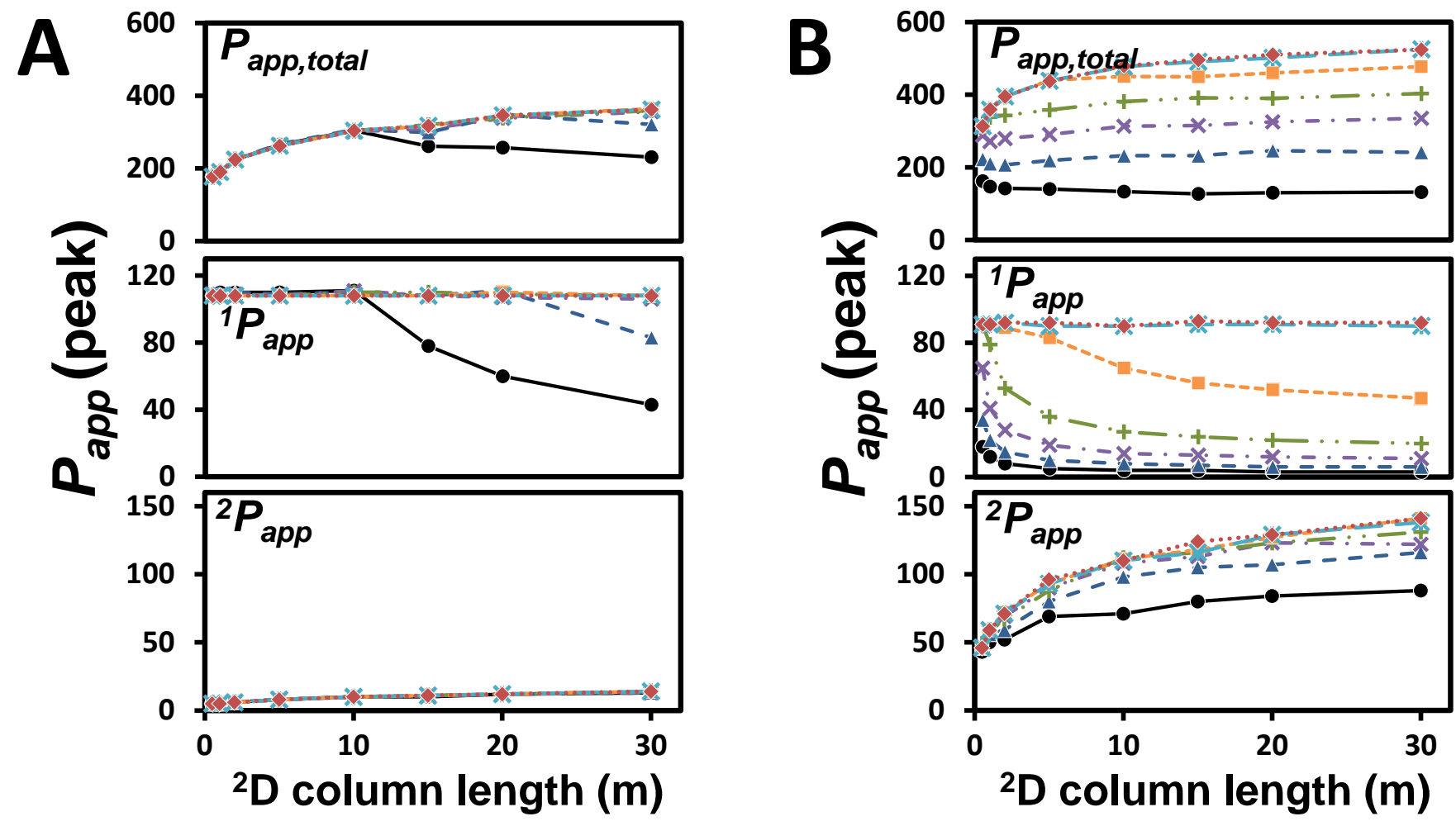

Figure S7. Plots of apparent number of separated peaks $\left(P_{a p p}\right)$ as functions of ${ }^{2} \mathrm{D}$ column length $\left({ }^{2} L\right)$ at different number of injections to complete the comprehensive analysis $\left(N_{i n j}=1,2,4,8,20,40,100\right.$ represented by $\bullet, \Delta, \mathrm{X},+, \square, *$ and $\bullet$, respectively) for simulated separation results on 5\%(phenyl)methylpolysiloxane (5ms) ×polyethyleneglycol (WAX) (A) and SLB-IL82×5ms (B) column sets. 


\section{Additional Table}

Table S1. List of the analytes with the corresponding LSER descriptors used in this study.

\begin{tabular}{|c|c|c|c|c|c|}
\hline Analytes & $\mathbf{E}$ & $\mathbf{S}$ & $\mathbf{A}$ & B & $\mathbf{L}$ \\
\hline (3-methyl-5-hexen-1-yn-1-yl)benzene & 0.099 & 0.120 & 0.060 & 0.019 & 0.089 \\
\hline 5-(4-pentyn-1-yl)indane & 0.099 & 0.120 & 0.060 & 0.019 & 0.089 \\
\hline (1-cyclohexen-1-ylethynyl)benzene & 0.099 & 0.120 & 0.060 & 0.019 & 0.089 \\
\hline (cyclohexylethynyl)benzene & 0.099 & 0.120 & 0.060 & 0.019 & 0.089 \\
\hline (1Z)-1-octen-3-yn-1-ylbenzene & 0.099 & 0.120 & 0.060 & 0.019 & 0.089 \\
\hline (1E)-1-octen-3-yn-1-ylbenzene & 0.099 & 0.120 & 0.060 & 0.019 & 0.089 \\
\hline 2-othynyl-4-methyl-1-[(2S)-4-penten-2-yl]benzene & 0.099 & 0.120 & 0.060 & 0.019 & 0.089 \\
\hline 1-(1-hexyn-1-yl)-2-vinylbenzene & 0.099 & 0.120 & 0.060 & 0.019 & 0.089 \\
\hline [3-(1-cyclohexen-1-yl)-2-propyn-1-yl]benzene & 0.099 & 0.120 & 0.060 & 0.019 & 0.089 \\
\hline $\begin{array}{l}\text { 1,3,5-trimethyl-2-[(1E)-1-penten-3-yn-1- } \\
\text { yl]benzene }\end{array}$ & 0.166 & 0.133 & 0.010 & 0.013 & 0.718 \\
\hline (5-methyl-3,4-octadien-6-yn-1-yl)benzene & 0.166 & 0.133 & 0.010 & 0.013 & 0.718 \\
\hline methanal & 0.220 & 0.620 & 0.000 & 0.330 & 0.730 \\
\hline ethanal & 0.208 & 0.670 & 0.000 & 0.450 & 1.230 \\
\hline 1-octen-5-yn-4-ylbenzene & 0.155 & 0.104 & 0.010 & 0.095 & 1.261 \\
\hline dimethylether & 0.190 & 0.360 & 0.010 & 0.435 & 1.391 \\
\hline 2-propanone & 0.179 & 0.700 & 0.400 & 0.490 & 1.696 \\
\hline acetone & 0.282 & 0.701 & 0.030 & 0.493 & 1.791 \\
\hline propanal & 0.196 & 0.650 & 0.000 & 0.450 & 1.815 \\
\hline furan & 0.543 & 0.638 & 0.020 & 0.193 & 2.177 \\
\hline cyclopropanpone & 0.391 & 0.790 & 0.040 & 0.474 & 2.199 \\
\hline butanal & 0.187 & 0.650 & 0.000 & 0.450 & 2.270 \\
\hline 1,4-nonadiyn-1-ylbenzene & 0.135 & 0.096 & 0.000 & 0.100 & 2.282 \\
\hline 2-butanone & 0.166 & 0.700 & 0.000 & 0.510 & 2.287 \\
\hline methylpropanone & 0.267 & 0.710 & 0.000 & 0.519 & 2.339 \\
\hline methoxymethylamine & 0.380 & 0.721 & 0.330 & 0.694 & 2.487 \\
\hline cyclobutenone & 0.438 & 0.874 & 0.020 & 0.471 & 2.539 \\
\hline cyclobutanone & 0.387 & 0.812 & 0.020 & 0.517 & 2.612 \\
\hline 2-pentanone & 0.143 & 0.680 & 0.000 & 0.510 & 2.755 \\
\hline heptyne & 0.134 & 0.100 & 0.000 & 0.098 & 2.763 \\
\hline pentanal & 0.163 & 0.650 & 0.000 & 0.450 & 2.770 \\
\hline ethylpropanone & 0.255 & 0.692 & 0.000 & 0.534 & 2.824 \\
\hline ethylketone & 0.265 & 0.679 & 0.000 & 0.537 & 2.860 \\
\hline isoheptene & 0.183 & 0.194 & 0.000 & 0.171 & 3.085 \\
\hline isooctene & 0.183 & 0.194 & 0.000 & 0.171 & 3.085 \\
\hline furanone & 0.547 & 1.021 & 0.020 & 0.509 & 3.097 \\
\hline pentanol & 0.317 & 0.473 & 0.370 & 0.512 & 3.110 \\
\hline isoheptane & 0.116 & 0.089 & 0.000 & 0.099 & 3.112 \\
\hline methylisobutylketone & 0.233 & 0.665 & 0.000 & 0.543 & 3.125 \\
\hline
\end{tabular}




\begin{tabular}{|c|c|c|c|c|c|}
\hline 1,6-heptadiene & 0.263 & 0.261 & 0.000 & 0.176 & 3.153 \\
\hline 1,3-heptadiene & 0.303 & 0.309 & 0.000 & 0.168 & 3.165 \\
\hline 1,4-heptadiene & 0.263 & 0.224 & 0.000 & 0.173 & 3.166 \\
\hline heptene & 0.189 & 0.200 & 0.000 & 0.163 & 3.167 \\
\hline 1,5-heptadiene & 0.288 & 0.223 & 0.000 & 0.176 & 3.170 \\
\hline [(3E)-3-Ethyl-3-hexen-1-yn-1-yl]benzene & 0.259 & 0.191 & 0.000 & 0.176 & 3.198 \\
\hline cyclopentenone & 0.506 & 0.890 & 0.020 & 0.517 & 3.201 \\
\hline cyclopentadienone & 0.678 & 0.897 & 0.030 & 0.484 & 3.205 \\
\hline cyclopentanone & 0.442 & 0.845 & 0.010 & 0.546 & 3.220 \\
\hline$n$-heptane & 0.135 & 0.097 & 0.000 & 0.102 & 3.251 \\
\hline octyne & 0.135 & 0.097 & 0.000 & 0.102 & 3.251 \\
\hline dimethylfuran & 0.647 & 0.768 & 0.010 & 0.306 & 3.260 \\
\hline 2-hexanone & 0.136 & 0.680 & 0.000 & 0.510 & 3.286 \\
\hline ethylbutanone & 0.254 & 0.676 & 0.000 & 0.536 & 3.319 \\
\hline methylpentanone & 0.247 & 0.693 & 0.000 & 0.536 & 3.326 \\
\hline 2-hexanol & 0.187 & 0.360 & 0.330 & 0.560 & 3.340 \\
\hline hexanal & 0.146 & 0.650 & 0.000 & 0.450 & 3.370 \\
\hline 2,2,4-trimethylpentane & 0.144 & 0.100 & 0.000 & 0.116 & 3.383 \\
\hline 2,2,4-trimethylpentene & 0.144 & 0.100 & 0.000 & 0.116 & 3.383 \\
\hline methylbenzene & 0.708 & 0.596 & 0.000 & 0.223 & 3.418 \\
\hline methylcyclohexane & 0.306 & 0.167 & 0.000 & 0.099 & 3.465 \\
\hline ethylcyclopentene & 0.386 & 0.231 & 0.000 & 0.182 & 3.573 \\
\hline diisopropylketone & 0.225 & 0.627 & 0.000 & 0.552 & 3.604 \\
\hline 1-hexanol & 0.210 & 0.420 & 0.370 & 0.480 & 3.610 \\
\hline 1,2,4-trimethylpentane & 0.141 & 0.083 & 0.000 & 0.100 & 3.635 \\
\hline 1,2,4-trimethylpentene & 0.270 & 0.189 & 0.000 & 0.194 & 3.648 \\
\hline isooctane & 0.129 & 0.094 & 0.000 & 0.102 & 3.656 \\
\hline cyclohexadienone & 0.697 & 0.895 & 0.010 & 0.528 & 3.662 \\
\hline octene & 0.188 & 0.200 & 0.000 & 0.167 & 3.670 \\
\hline cyclohexenone & 0.518 & 0.887 & 0.000 & 0.549 & 3.679 \\
\hline 1,5-octadiene & 0.267 & 0.217 & 0.000 & 0.172 & 3.698 \\
\hline 1,6-octadiene & 0.268 & 0.210 & 0.000 & 0.173 & 3.699 \\
\hline 1,4-octadiene & 0.255 & 0.244 & 0.000 & 0.174 & 3.711 \\
\hline cycloheptene & 0.428 & 0.255 & 0.000 & 0.176 & 3.712 \\
\hline $1,2,3$-trimethylpentane & 0.145 & 0.086 & 0.000 & 0.103 & 3.713 \\
\hline $1,2,3$-trimethylpentene & 0.261 & 0.181 & 0.000 & 0.192 & 3.721 \\
\hline 1,7-octadiene & 0.262 & 0.263 & 0.000 & 0.173 & 3.722 \\
\hline 1,3-octadiene & 0.297 & 0.352 & 0.000 & 0.167 & 3.725 \\
\hline 1-methyl-4-methylcyclohexane & 0.220 & 0.203 & 0.000 & 0.085 & 3.728 \\
\hline cyclohexanone & 0.457 & 0.820 & 0.000 & 0.570 & 3.732 \\
\hline cycloheptane & 0.412 & 0.173 & 0.000 & 0.101 & 3.746 \\
\hline propylketone & 0.240 & 0.677 & 0.000 & 0.541 & 3.757 \\
\hline 2-heptanone & 0.123 & 0.680 & 0.000 & 0.510 & 3.760 \\
\hline
\end{tabular}




\begin{tabular}{|c|c|c|c|c|c|}
\hline cycloheptatrienone & 0.495 & 0.748 & 0.010 & 0.463 & 3.767 \\
\hline$n$-octane & 0.141 & 0.099 & 0.000 & 0.103 & 3.781 \\
\hline nonyne & 0.141 & 0.099 & 0.000 & 0.103 & 3.781 \\
\hline ethylpentanone & 0.238 & 0.685 & 0.000 & 0.539 & 3.784 \\
\hline 1-methyl-3-methylcyclohexane & 0.242 & 0.204 & 0.000 & 0.087 & 3.801 \\
\hline$N$-isopropyl-2-methoxyethylamine & 0.304 & 0.650 & 0.150 & 0.878 & 3.805 \\
\hline 1-methyl-2-methylcyclohexane & 0.261 & 0.203 & 0.000 & 0.082 & 3.808 \\
\hline 1-methyl-1-methylcyclohexane & 0.304 & 0.207 & 0.000 & 0.101 & 3.858 \\
\hline heptanal & 0.140 & 0.650 & 0.000 & 0.450 & 3.860 \\
\hline dimethylfuranone & 0.634 & 0.959 & 0.020 & 0.587 & 3.873 \\
\hline$p$-xylene & 0.690 & 0.567 & 0.000 & 0.229 & 3.888 \\
\hline$m$-xylene & 0.701 & 0.570 & 0.000 & 0.230 & 3.895 \\
\hline propylcyclopentene & 0.367 & 0.218 & 0.000 & 0.188 & 3.909 \\
\hline propylfuran & 0.637 & 0.774 & 0.000 & 0.323 & 3.910 \\
\hline ethylbenzene & 0.721 & 0.593 & 0.000 & 0.235 & 3.925 \\
\hline ethylcyclohexane & 0.319 & 0.158 & 0.000 & 0.107 & 3.932 \\
\hline$o$-xylene & 0.716 & 0.588 & 0.000 & 0.230 & 3.959 \\
\hline dimethylbenzene & 0.716 & 0.588 & 0.000 & 0.230 & 3.959 \\
\hline dibutylether & 0.170 & 0.329 & 0.000 & 0.484 & 4.011 \\
\hline methylcycloheptane & 0.295 & 0.164 & 0.000 & 0.111 & 4.018 \\
\hline vinylbenzene & 0.878 & 0.697 & 0.000 & 0.247 & 4.028 \\
\hline styrene & 0.878 & 0.697 & 0.000 & 0.247 & 4.028 \\
\hline 2,2,4-trimethylhexane & 0.155 & 0.093 & 0.000 & 0.111 & 4.063 \\
\hline isopropoxypropylamine & 0.331 & 0.687 & 0.190 & 0.830 & 4.097 \\
\hline 2-heptanol & 0.188 & 0.360 & 0.330 & 0.560 & 4.115 \\
\hline 1-heptanol & 0.211 & 0.420 & 0.370 & 0.480 & 4.115 \\
\hline isononane & 0.134 & 0.092 & 0.000 & 0.105 & 4.133 \\
\hline 1,5-nonadiene & 0.273 & 0.211 & 0.000 & 0.181 & 4.168 \\
\hline cyclooctene & 0.466 & 0.263 & 0.000 & 0.180 & 4.168 \\
\hline 1,2,3-trimethylhexane & 0.154 & 0.094 & 0.000 & 0.117 & 4.171 \\
\hline 1,6-nonadiene & 0.265 & 0.209 & 0.000 & 0.184 & 4.174 \\
\hline 1,2,3-trimethylhexene & 0.268 & 0.163 & 0.000 & 0.193 & 4.176 \\
\hline 1,4-nonadiene & 0.262 & 0.212 & 0.000 & 0.181 & 4.191 \\
\hline 1,2,4-trimethylhexane & 0.148 & 0.091 & 0.000 & 0.109 & 4.193 \\
\hline 1,3-nonadiene & 0.299 & 0.305 & 0.000 & 0.178 & 4.193 \\
\hline 1,2,4-trimethylhexene & 0.270 & 0.186 & 0.000 & 0.197 & 4.195 \\
\hline cycloheptenone & 0.486 & 0.814 & 0.000 & 0.551 & 4.204 \\
\hline cycloheptanone & 0.440 & 0.743 & 0.000 & 0.561 & 4.220 \\
\hline nonene & 0.204 & 0.200 & 0.000 & 0.170 & 4.221 \\
\hline cycloheptadienone & 0.637 & 0.827 & 0.000 & 0.537 & 4.225 \\
\hline cyclooctane & 0.448 & 0.172 & 0.000 & 0.105 & 4.235 \\
\hline n-sec-butyl-2-methoxyethylamine & 0.289 & 0.654 & 0.140 & 0.869 & 4.237 \\
\hline$n$-nonane & 0.148 & 0.104 & 0.000 & 0.106 & 4.243 \\
\hline
\end{tabular}




\begin{tabular}{|c|c|c|c|c|c|}
\hline decyne & 0.148 & 0.104 & 0.000 & 0.106 & 4.243 \\
\hline 2-octanone & 0.108 & 0.680 & 0.000 & 0.510 & 4.257 \\
\hline propylpentanone & 0.252 & 0.688 & 0.000 & 0.538 & 4.313 \\
\hline ethylhexanone & 0.252 & 0.688 & 0.000 & 0.538 & 4.313 \\
\hline isopropylbenzene & 0.725 & 0.585 & 0.000 & 0.240 & 4.318 \\
\hline 1,1,2-trimethylcyclohexane & 0.410 & 0.238 & 0.000 & 0.122 & 4.331 \\
\hline octanal & 0.137 & 0.650 & 0.000 & 0.450 & 4.361 \\
\hline 1-ethyl-4-methylcyclohexane & 0.299 & 0.214 & 0.000 & 0.122 & 4.371 \\
\hline isopropylcyclohexane & 0.341 & 0.174 & 0.000 & 0.136 & 4.383 \\
\hline isopropylcycloheptane & 0.341 & 0.174 & 0.000 & 0.136 & 4.383 \\
\hline propylbenzene & 0.719 & 0.616 & 0.000 & 0.249 & 4.386 \\
\hline 1-ethyl-1-methylcyclohexane & 0.378 & 0.202 & 0.000 & 0.128 & 4.407 \\
\hline 1-methyl-4-ethylbenzene & 0.728 & 0.565 & 0.000 & 0.253 & 4.408 \\
\hline 1-ethyl-3-methylcyclohexane & 0.301 & 0.215 & 0.000 & 0.127 & 4.409 \\
\hline 1-methyl-3-ethylbenzene & 0.734 & 0.570 & 0.000 & 0.250 & 4.411 \\
\hline 1,2,4-trimethylcyclohexane & 0.346 & 0.277 & 0.000 & 0.136 & 4.414 \\
\hline dimethylcycloheptane & 0.329 & 0.156 & 0.000 & 0.137 & 4.414 \\
\hline propylcyclohexane & 0.346 & 0.193 & 0.000 & 0.143 & 4.421 \\
\hline butylcyclopentene & 0.394 & 0.218 & 0.000 & 0.207 & 4.424 \\
\hline 1,2,3-trimethylcyclohexane & 0.418 & 0.275 & 0.000 & 0.135 & 4.427 \\
\hline 1-ethyl-2-methylcyclohexane & 0.334 & 0.203 & 0.000 & 0.123 & 4.431 \\
\hline propylcyclohexene & 0.400 & 0.223 & 0.000 & 0.216 & 4.434 \\
\hline 1-methyl-2-ethylbenzene & 0.751 & 0.585 & 0.000 & 0.251 & 4.435 \\
\hline 1,3,5-trimethylcyclohexane & 0.332 & 0.278 & 0.000 & 0.125 & 4.456 \\
\hline ethylcycloheptane & 0.337 & 0.166 & 0.000 & 0.127 & 4.459 \\
\hline 1,2,4-trimethylbenzene & 0.742 & 0.590 & 0.000 & 0.260 & 4.466 \\
\hline ethylcycloheptene & 0.408 & 0.233 & 0.000 & 0.211 & 4.474 \\
\hline 2,2,4-trimethylheptane & 0.166 & 0.104 & 0.000 & 0.131 & 4.523 \\
\hline 1,2,3-trimethylbenzene & 0.762 & 0.613 & 0.000 & 0.259 & 4.525 \\
\hline butylfuran & 0.640 & 0.800 & 0.000 & 0.347 & 4.535 \\
\hline diisobutylketone & 0.225 & 0.643 & 0.000 & 0.551 & 4.550 \\
\hline methylcyclooctane & 0.378 & 0.176 & 0.000 & 0.128 & 4.600 \\
\hline acetophenone & 0.882 & 0.994 & 0.000 & 0.516 & 4.605 \\
\hline 2-octanol & 0.158 & 0.360 & 0.330 & 0.560 & 4.619 \\
\hline 1-octanol & 0.199 & 0.420 & 0.370 & 0.480 & 4.619 \\
\hline methylcyclooctene & 0.441 & 0.250 & 0.000 & 0.206 & 4.642 \\
\hline 2-methylanisole & 0.827 & 0.795 & 0.000 & 0.340 & 4.654 \\
\hline cyclopentylpropanone & 0.416 & 0.764 & 0.000 & 0.585 & 4.661 \\
\hline 1,5-decadiene & 0.276 & 0.212 & 0.000 & 0.183 & 4.676 \\
\hline 1,6-decadiene & 0.276 & 0.209 & 0.000 & 0.190 & 4.676 \\
\hline 1,3-decadiene & 0.307 & 0.362 & 0.000 & 0.180 & 4.678 \\
\hline 1,4-decadiene & 0.272 & 0.217 & 0.000 & 0.187 & 4.691 \\
\hline indan & 0.888 & 0.651 & 0.000 & 0.219 & 4.694 \\
\hline
\end{tabular}




\begin{tabular}{|c|c|c|c|c|c|}
\hline decene & 0.208 & 0.216 & 0.000 & 0.167 & 4.706 \\
\hline 2,4-dimethyl-3-heptanone & 0.271 & 0.667 & 0.000 & 0.572 & 4.708 \\
\hline isodecane & 0.147 & 0.100 & 0.000 & 0.115 & 4.725 \\
\hline 1,2,3-trimethylheptane & 0.178 & 0.096 & 0.000 & 0.129 & 4.725 \\
\hline 2-nonanone & 0.113 & 0.680 & 0.000 & 0.510 & 4.735 \\
\hline 1,2,3-trimethylheptene & 0.231 & 0.201 & 0.000 & 0.217 & 4.755 \\
\hline indene & 1.031 & 0.817 & 0.010 & 0.239 & 4.783 \\
\hline isopentylcyclopentene & 0.394 & 0.218 & 0.000 & 0.209 & 4.799 \\
\hline 1,2,4-trimethylheptane & 0.169 & 0.101 & 0.000 & 0.129 & 4.800 \\
\hline$n$-decane & 0.148 & 0.109 & 0.000 & 0.105 & 4.805 \\
\hline undecyne & 0.148 & 0.109 & 0.000 & 0.105 & 4.805 \\
\hline 1,2-dimethyl-4-ethylbenzene & 0.737 & 0.586 & 0.000 & 0.261 & 4.809 \\
\hline 1-methyl-4-propylcyclohexane & 0.324 & 0.248 & 0.000 & 0.112 & 4.812 \\
\hline butylketone & 0.243 & 0.703 & 0.000 & 0.534 & 4.825 \\
\hline cyclononene & 0.497 & 0.260 & 0.000 & 0.196 & 4.828 \\
\hline 1,4-diethylbenzene & 0.723 & 0.551 & 0.000 & 0.246 & 4.834 \\
\hline butylpentanone & 0.246 & 0.702 & 0.000 & 0.534 & 4.835 \\
\hline nonanal & 0.150 & 0.650 & 0.000 & 0.450 & 4.856 \\
\hline 1,2-dimethyl-3-ethylbenzene & 0.751 & 0.585 & 0.000 & 0.258 & 4.862 \\
\hline cyclononane & 0.488 & 0.187 & 0.000 & 0.112 & 4.867 \\
\hline 1-methyl-1-propylcyclohexane & 0.411 & 0.220 & 0.000 & 0.128 & 4.881 \\
\hline 1-methyl-2-propylbenzene & 0.735 & 0.588 & 0.000 & 0.250 & 4.887 \\
\hline butylcyclohexane & 0.389 & 0.205 & 0.000 & 0.122 & 4.889 \\
\hline butylbenzene & 0.737 & 0.635 & 0.000 & 0.250 & 4.890 \\
\hline 1-methyl-2-propylcyclohexane & 0.370 & 0.256 & 0.000 & 0.114 & 4.891 \\
\hline 1-methyl-3-propyl-cyclohexane & 0.333 & 0.244 & 0.000 & 0.109 & 4.892 \\
\hline 1,2,4,5-tetramethylbenzene & 0.746 & 0.595 & 0.000 & 0.257 & 4.894 \\
\hline isobutylcyclohexane & 0.353 & 0.210 & 0.000 & 0.131 & 4.895 \\
\hline 1,2,3,4-tetramethylbenzene & 0.766 & 0.604 & 0.000 & 0.259 & 4.900 \\
\hline 1,3-diethylbenzene & 0.719 & 0.553 & 0.000 & 0.244 & 4.902 \\
\hline cyclooctanone & 0.499 & 0.748 & 0.000 & 0.565 & 4.908 \\
\hline $\begin{array}{l}\text { 1-Methyl-2-[(1E)-5-methyl-1-hexen-3-yn-1- } \\
\text { yl]benzene }\end{array}$ & 0.884 & 0.729 & 0.000 & 0.247 & 4.912 \\
\hline butylcyclohexene & 0.455 & 0.237 & 0.000 & 0.213 & 4.923 \\
\hline cyclooctatrienone & 0.729 & 0.883 & 0.010 & 0.528 & 4.954 \\
\hline 2,2,4-trimethyloctane & 0.180 & 0.106 & 0.000 & 0.144 & 4.971 \\
\hline 1,2-diethylbenzene & 0.741 & 0.568 & 0.000 & 0.245 & 4.976 \\
\hline propylcycloheptene & 0.428 & 0.240 & 0.000 & 0.213 & 4.980 \\
\hline benzylmethylketone & 0.839 & 0.940 & 0.000 & 0.639 & 4.980 \\
\hline divinylbenzene & 0.935 & 0.828 & 0.000 & 0.262 & 5.004 \\
\hline methylacetophenone & 0.896 & 0.995 & 0.000 & 0.532 & 5.115 \\
\hline 2-nonanol & 0.168 & 0.360 & 0.330 & 0.560 & 5.120 \\
\hline 1-nonanol & 0.193 & 0.420 & 0.370 & 0.480 & 5.120 \\
\hline
\end{tabular}




\begin{tabular}{|c|c|c|c|c|c|}
\hline$o$-acetyltoluene & 0.883 & 0.993 & 0.000 & 0.533 & 5.123 \\
\hline decalin & 0.537 & 0.284 & 0.000 & 0.063 & 5.160 \\
\hline ethylanisole & 0.855 & 0.873 & 0.000 & 0.394 & 5.162 \\
\hline ethylcyclooctane & 0.370 & 0.176 & 0.000 & 0.117 & 5.164 \\
\hline$m$-acetyltoluene & 0.886 & 0.998 & 0.000 & 0.526 & 5.165 \\
\hline ethylcyclooctene & 0.472 & 0.218 & 0.000 & 0.194 & 5.166 \\
\hline cyclopentyl-2-butanone & 0.424 & 0.805 & 0.000 & 0.575 & 5.176 \\
\hline 1,6-undecadiene & 0.268 & 0.224 & 0.000 & 0.194 & 5.183 \\
\hline 1,5-undecadiene & 0.264 & 0.264 & 0.000 & 0.195 & 5.184 \\
\hline 1,4-undecadiene & 0.262 & 0.264 & 0.000 & 0.199 & 5.188 \\
\hline 1,3-undecadiene & 0.303 & 0.390 & 0.000 & 0.197 & 5.190 \\
\hline isoundecane & 0.155 & 0.103 & 0.000 & 0.120 & 5.201 \\
\hline undecene & 0.211 & 0.229 & 0.000 & 0.174 & 5.208 \\
\hline naphthalene & 1.231 & 0.888 & 0.000 & 0.264 & 5.210 \\
\hline tetralin & 0.915 & 0.674 & 0.000 & 0.238 & 5.211 \\
\hline methylindan & 0.967 & 0.733 & 0.020 & 0.243 & 5.217 \\
\hline 2,2,4-trimethyloctene & 0.254 & 0.207 & 0.000 & 0.221 & 5.230 \\
\hline 2-decanone & 0.108 & 0.680 & 0.000 & 0.510 & 5.245 \\
\hline 1,2,3-trimethyl-5-ethylbenzene & 0.777 & 0.616 & 0.000 & 0.273 & 5.284 \\
\hline$n$-undecane & 0.152 & 0.114 & 0.000 & 0.105 & 5.303 \\
\hline dodecyne & 0.152 & 0.114 & 0.000 & 0.105 & 5.303 \\
\hline methylindene & 1.089 & 0.910 & 0.030 & 0.254 & 5.331 \\
\hline 1,2,3-trimethyl-4-ethylbenzene & 0.791 & 0.633 & 0.000 & 0.271 & 5.353 \\
\hline methylcyclononane & 0.412 & 0.193 & 0.000 & 0.118 & 5.358 \\
\hline methylcyclononene & 0.461 & 0.269 & 0.000 & 0.209 & 5.364 \\
\hline 1-ethynyl-2-(3-methyl-2-buten-1-yl)benzene & 0.795 & 0.624 & 0.000 & 0.262 & 5.385 \\
\hline cyclodecane & 0.505 & 0.187 & 0.000 & 0.102 & 5.405 \\
\hline 1-methyl-2-butylbenzene & 0.740 & 0.632 & 0.000 & 0.257 & 5.421 \\
\hline 1,2-dimethyl-3-propylbenzene & 0.754 & 0.629 & 0.000 & 0.270 & 5.421 \\
\hline 1-methyl-2-butylcyclohexane & 0.417 & 0.294 & 0.000 & 0.098 & 5.426 \\
\hline cyclodecene & 0.511 & 0.267 & 0.000 & 0.186 & 5.433 \\
\hline pentylbenzene & 0.740 & 0.660 & 0.000 & 0.256 & 5.435 \\
\hline isopentylcyclohexane & 0.368 & 0.252 & 0.000 & 0.127 & 5.440 \\
\hline pentylcyclohexene & 0.445 & 0.280 & 0.000 & 0.210 & 5.440 \\
\hline 1,2-dimethyl-4-propylbenzene & 0.746 & 0.629 & 0.000 & 0.272 & 5.460 \\
\hline 1-ethyl-4-propylcyclohexane & 0.368 & 0.290 & 0.000 & 0.106 & 5.463 \\
\hline pentamethylbenzene & 0.861 & 0.663 & 0.000 & 0.276 & 5.465 \\
\hline 1-ethyl-2-propylbenzene & 0.739 & 0.615 & 0.000 & 0.250 & 5.466 \\
\hline methylbutylcyclohexane & 0.419 & 0.290 & 0.000 & 0.124 & 5.474 \\
\hline isopropylethylbenzene & 0.750 & 0.595 & 0.000 & 0.263 & 5.488 \\
\hline 1,2,3,4-tetrahydroquinoline (THQ) & 1.056 & 0.943 & 0.220 & 0.554 & 5.508 \\
\hline hexylcyclopentene & 0.440 & 0.272 & 0.000 & 0.202 & 5.532 \\
\hline pentylcyclohexane & 0.421 & 0.252 & 0.000 & 0.120 & 5.544 \\
\hline
\end{tabular}




\begin{tabular}{|c|c|c|c|c|c|}
\hline benzylethylketone & 0.836 & 1.014 & 0.000 & 0.570 & 5.560 \\
\hline$p$-ethylacetophenone & 0.895 & 1.050 & 0.000 & 0.550 & 5.562 \\
\hline$m$-ethylacetophenone & 0.883 & 1.043 & 0.000 & 0.549 & 5.573 \\
\hline cyclononanone & 0.501 & 0.773 & 0.000 & 0.562 & 5.576 \\
\hline 1-methyl-1-methylindan & 1.017 & 0.692 & 0.000 & 0.230 & 5.586 \\
\hline 1-cyclohexylbutan-2-one & 0.483 & 0.803 & 0.000 & 0.588 & 5.600 \\
\hline$o$-ethylacetophenone & 0.881 & 1.055 & 0.000 & 0.552 & 5.605 \\
\hline 1-decanol & 0.191 & 0.420 & 0.370 & 0.480 & 5.628 \\
\hline butylcycloheptene & 0.452 & 0.259 & 0.000 & 0.199 & 5.641 \\
\hline 1-ethyl-2-propylcyclohexane & 0.445 & 0.305 & 0.000 & 0.112 & 5.646 \\
\hline 1,5-dodecadiene & 0.283 & 0.242 & 0.000 & 0.188 & 5.665 \\
\hline 1,6-dodecadiene & 0.283 & 0.523 & 0.000 & 0.190 & 5.665 \\
\hline 1,4-dodecadiene & 0.278 & 0.288 & 0.000 & 0.207 & 5.673 \\
\hline butylcycloheptane & 0.382 & 0.196 & 0.000 & 0.114 & 5.674 \\
\hline 1,2-dimethylindan & 1.084 & 0.689 & 0.000 & 0.252 & 5.688 \\
\hline 1,3-dodecadiene & 0.317 & 0.356 & 0.000 & 0.189 & 5.690 \\
\hline diisopentylketone & 0.263 & 0.697 & 0.000 & 0.629 & 5.694 \\
\hline dodecene & 0.226 & 0.249 & 0.000 & 0.180 & 5.698 \\
\hline isododecane & 0.174 & 0.121 & 0.000 & 0.123 & 5.718 \\
\hline 1,1-dimethylindene & 1.143 & 0.936 & 0.000 & 0.242 & 5.728 \\
\hline 2-undecanone & 0.101 & 0.680 & 0.000 & 0.510 & 5.732 \\
\hline methylcyclodecane & 0.424 & 0.220 & 0.000 & 0.123 & 5.751 \\
\hline pentylketone & 0.262 & 0.744 & 0.000 & 0.537 & 5.764 \\
\hline$n$-dodecane & 0.171 & 0.129 & 0.000 & 0.108 & 5.777 \\
\hline tridecyne & 0.171 & 0.129 & 0.000 & 0.108 & 5.777 \\
\hline ethylindan & 1.016 & 0.792 & 0.000 & 0.257 & 5.797 \\
\hline methyltetralin & 1.059 & 0.787 & 0.000 & 0.254 & 5.806 \\
\hline 2-methylnaphthalene & 1.298 & 0.902 & 0.000 & 0.297 & 5.834 \\
\hline cycloundecene & 0.537 & 0.293 & 0.000 & 0.186 & 5.850 \\
\hline propylcyclooctene & 0.464 & 0.265 & 0.000 & 0.201 & 5.861 \\
\hline 1-methylnaphthalene & 1.309 & 0.927 & 0.000 & 0.294 & 5.871 \\
\hline hexylbenzene & 0.751 & 0.690 & 0.000 & 0.255 & 5.877 \\
\hline cyclodecanone & 0.514 & 0.788 & 0.000 & 0.568 & 5.890 \\
\hline 1-methylene-1,2,3,4-tetrahydronaphthalene & 1.153 & 0.906 & 0.000 & 0.260 & 5.908 \\
\hline cycloundecane & 0.529 & 0.215 & 0.000 & 0.106 & 5.911 \\
\hline heptylcyclopentene & 0.452 & 0.304 & 0.000 & 0.204 & 5.917 \\
\hline hexylcyclohexane & 0.437 & 0.294 & 0.000 & 0.115 & 5.958 \\
\hline 1-methyl-2-pentylbenzene & 0.759 & 0.681 & 0.000 & 0.250 & 5.964 \\
\hline benzylpropylketone & 0.834 & 1.056 & 0.000 & 0.573 & 5.982 \\
\hline hexylcyclohexene & 0.508 & 0.321 & 0.000 & 0.203 & 5.988 \\
\hline isohexylcyclohexane & 0.398 & 0.340 & 0.000 & 0.109 & 5.992 \\
\hline 1-methoxyindan & 1.068 & 1.001 & 0.010 & 0.555 & 6.023 \\
\hline$p$-propylacetophenone & 0.887 & 1.059 & 0.000 & 0.551 & 6.041 \\
\hline
\end{tabular}




\begin{tabular}{|c|c|c|c|c|c|}
\hline 1,6-tridecadiene & 0.295 & 0.302 & 0.000 & 0.216 & 6.043 \\
\hline 1,4-tridecadiene & 0.293 & 0.265 & 0.000 & 0.193 & 6.050 \\
\hline 1,3-tridecadiene & 0.334 & 0.376 & 0.000 & 0.185 & 6.052 \\
\hline 1,5 -tridecadiene & 0.296 & 0.308 & 0.000 & 0.213 & 6.053 \\
\hline$o$-propylacetophenone & 0.897 & 1.084 & 0.000 & 0.557 & 6.085 \\
\hline methyldecalin & 0.723 & 0.432 & 0.000 & 0.130 & 6.092 \\
\hline isotridecane & 0.171 & 0.134 & 0.000 & 0.125 & 6.122 \\
\hline tridecene & 0.266 & 0.221 & 0.000 & 0.185 & 6.124 \\
\hline 1-undecanol & 0.181 & 0.420 & 0.370 & 0.480 & 6.128 \\
\hline 1,2-dimethyl-4-butylbenzene & 0.769 & 0.665 & 0.000 & 0.274 & 6.131 \\
\hline 1-methyl-3-pentylcyclohexane & 0.414 & 0.329 & 0.000 & 0.100 & 6.137 \\
\hline tetralone & 1.213 & 1.162 & 0.010 & 0.542 & 6.141 \\
\hline cyclohexylbenzene & 0.950 & 0.724 & 0.000 & 0.232 & 6.162 \\
\hline biphenyl & 1.221 & 0.972 & 0.000 & 0.308 & 6.165 \\
\hline cyclododecene & 0.557 & 0.309 & 0.000 & 0.180 & 6.166 \\
\hline (2,4-cyclopentadien-1-ylidenemethyl)benzene & 1.176 & 0.880 & 0.000 & 0.271 & 6.167 \\
\hline 2,6-dimethylnaphthalene & 1.381 & 0.877 & 0.000 & 0.314 & 6.215 \\
\hline cycloheptyl-3-butanone & 0.451 & 0.798 & 0.000 & 0.593 & 6.240 \\
\hline 1-methyl-1-pentylcyclohexane & 0.474 & 0.305 & 0.000 & 0.116 & 6.241 \\
\hline 2-vinylnaphthalene & 1.384 & 0.954 & 0.000 & 0.293 & 6.246 \\
\hline 1-propyl-2-propylbenzene & 0.782 & 0.658 & 0.000 & 0.251 & 6.248 \\
\hline 1,2-dimethyl-3-butylbenzene & 0.786 & 0.675 & 0.000 & 0.274 & 6.249 \\
\hline 1-ethyl-2-butylbenzene & 0.765 & 0.662 & 0.000 & 0.247 & 6.251 \\
\hline propylindan & 1.087 & 0.814 & 0.010 & 0.245 & 6.255 \\
\hline 2-ethylnaphthalene & 1.361 & 0.935 & 0.000 & 0.287 & 6.256 \\
\hline cyclododecane & 0.552 & 0.237 & 0.000 & 0.097 & 6.264 \\
\hline 1-methyl-2-pentylcyclohexane & 0.447 & 0.336 & 0.000 & 0.100 & 6.264 \\
\hline 1-ethylnaphthalene & 1.391 & 0.916 & 0.000 & 0.287 & 6.292 \\
\hline$n$-tridecane & 0.187 & 0.146 & 0.000 & 0.109 & 6.293 \\
\hline tetradecyne & 0.187 & 0.146 & 0.000 & 0.109 & 6.293 \\
\hline 1-vinylnaphthalene & 1.409 & 0.958 & 0.000 & 0.292 & 6.301 \\
\hline heptylbenzene & 0.766 & 0.741 & 0.000 & 0.256 & 6.313 \\
\hline 5-methyl-1-phenylhexa-1,3,4-triene & 0.963 & 0.873 & 0.000 & 0.258 & 6.341 \\
\hline 1,6-dimethylnaphthalene & 1.402 & 0.948 & 0.000 & 0.291 & 6.364 \\
\hline $1,2,4$-triethylbenzene & 0.814 & 0.665 & 0.000 & 0.264 & 6.370 \\
\hline 1-ethyl-3-butylcyclohexane & 0.423 & 0.344 & 0.000 & 0.109 & 6.374 \\
\hline ethylbutylcyclohexane & 0.493 & 0.352 & 0.000 & 0.114 & 6.401 \\
\hline 1,2,4-triethylcyclohexane & 0.532 & 0.440 & 0.000 & 0.127 & 6.440 \\
\hline butylcyclooctane & 0.410 & 0.226 & 0.000 & 0.115 & 6.465 \\
\hline ethyltetralin & 1.155 & 0.842 & 0.000 & 0.240 & 6.483 \\
\hline 1,2,3-triethylbenzene & 0.859 & 0.680 & 0.000 & 0.261 & 6.484 \\
\hline 1,2-dimethylnaphthalene & 1.447 & 0.990 & 0.000 & 0.301 & 6.515 \\
\hline ethylcyclodecane & 0.430 & 0.237 & 0.000 & 0.111 & 6.574 \\
\hline
\end{tabular}




\begin{tabular}{|c|c|c|c|c|c|}
\hline hexylketone & 0.272 & 0.775 & 0.000 & 0.561 & 6.575 \\
\hline$p$-butylacetophenone & 0.890 & 1.090 & 0.000 & 0.558 & 6.605 \\
\hline 1,3-tetradecadiene & 0.343 & 0.471 & 0.000 & 0.230 & 6.607 \\
\hline 1,4-tetradecadiene & 0.322 & 0.393 & 0.000 & 0.232 & 6.609 \\
\hline 1,5-tetradecadiene & 0.300 & 0.315 & 0.000 & 0.233 & 6.610 \\
\hline 1,6-tetradecadiene & 0.297 & 0.310 & 0.000 & 0.236 & 6.610 \\
\hline octylcyclopentene & 0.480 & 0.369 & 0.000 & 0.192 & 6.620 \\
\hline$o$-butylacetophenone & 0.908 & 1.112 & 0.000 & 0.573 & 6.632 \\
\hline acenaphthene & 1.540 & 1.122 & 0.000 & 0.210 & 6.637 \\
\hline tetradecene & 0.247 & 0.268 & 0.000 & 0.223 & 6.655 \\
\hline isotetradecane & 0.183 & 0.140 & 0.000 & 0.122 & 6.664 \\
\hline 1,2-dimethyltetralin & 1.257 & 0.743 & 0.000 & 0.236 & 6.679 \\
\hline heptylcyclohexene & 0.505 & 0.377 & 0.000 & 0.191 & 6.683 \\
\hline 1,2,3,4-tetramethyl-5-ethylbenzene & 0.906 & 0.706 & 0.000 & 0.287 & 6.688 \\
\hline 1,2-dimethyl-3-pentylbenzene & 0.782 & 0.683 & 0.000 & 0.265 & 6.697 \\
\hline acenaphthylene & 1.540 & 1.122 & 0.000 & 0.210 & 6.706 \\
\hline heptylcyclohexane & 0.443 & 0.344 & 0.000 & 0.116 & 6.707 \\
\hline 1,5-dimethyl-1,2,3,4-tetrahydronaphthalene & 1.254 & 0.745 & 0.000 & 0.222 & 6.715 \\
\hline 1-methyl-1-ethylindan & 1.099 & 0.720 & 0.000 & 0.219 & 6.716 \\
\hline 5,8-dimethyl-1,2,3,4-tetrahydronaphthalene & 1.236 & 0.802 & 0.000 & 0.222 & 6.725 \\
\hline$n$-tetradecane & 0.199 & 0.144 & 0.000 & 0.106 & 6.744 \\
\hline pentadecyne & 0.199 & 0.144 & 0.000 & 0.106 & 6.744 \\
\hline 1,5-pentadecadiene & 0.199 & 0.144 & 0.000 & 0.106 & 6.744 \\
\hline 1,6-pentadecadiene & 0.199 & 0.144 & 0.000 & 0.106 & 6.744 \\
\hline 1,1-dimethyltetralin & 1.144 & 0.738 & 0.000 & 0.206 & 6.752 \\
\hline 1,2,3-triethylcyclohexane & 0.601 & 0.434 & 0.000 & 0.132 & 6.754 \\
\hline (1Z)-1,2,3-octatrien-1-ylbenzene & 0.952 & 0.854 & 0.000 & 0.264 & 6.756 \\
\hline 2-methylbiphenyl & 1.383 & 1.009 & 0.000 & 0.321 & 6.773 \\
\hline 1-methyl-2-hexylbenzene & 0.782 & 0.734 & 0.000 & 0.253 & 6.781 \\
\hline butylindan & 1.151 & 0.885 & 0.008 & 0.241 & 6.807 \\
\hline butylatedhydroxyanisole & 0.985 & 1.150 & 0.430 & 0.583 & 6.819 \\
\hline 1-methyl-4-hexylcyclohexane & 0.392 & 0.366 & 0.000 & 0.097 & 6.851 \\
\hline 1-ethyl-2-pentylbenzene & 0.808 & 0.707 & 0.000 & 0.246 & 6.852 \\
\hline 3-methylbiphenyl & 1.375 & 1.023 & 0.000 & 0.324 & 6.854 \\
\hline 4-methylbiphenyl & 1.365 & 1.020 & 0.000 & 0.326 & 6.855 \\
\hline 1-propyl-2-butylbenzene & 0.822 & 0.707 & 0.000 & 0.246 & 6.859 \\
\hline cyclotridecane & 0.565 & 0.269 & 0.000 & 0.097 & 6.912 \\
\hline fluorene & 1.588 & 1.060 & 0.000 & 0.250 & 6.922 \\
\hline cyclotridecene & 0.568 & 0.334 & 0.000 & 0.181 & 6.945 \\
\hline methylhexylcyclohexane & 0.470 & 0.379 & 0.000 & 0.115 & 6.974 \\
\hline ethyldecalin & 0.933 & 0.483 & 0.000 & 0.130 & 6.974 \\
\hline 1-(3-buten-1-yl)-1h-indene & 1.312 & 1.054 & 0.000 & 0.295 & 7.029 \\
\hline 2-propylnaphthalene & 1.380 & 1.080 & 0.000 & 0.302 & 7.031 \\
\hline
\end{tabular}




\begin{tabular}{|c|c|c|c|c|c|}
\hline 3-methyl-7-phenylhepta-1,3,4-triene & 0.876 & 0.863 & 0.000 & 0.298 & 7.045 \\
\hline octylbenzene & 0.791 & 0.795 & 0.000 & 0.259 & 7.058 \\
\hline (1E)-1-octen-1-ylbenzene & 0.894 & 0.965 & 0.000 & 0.277 & 7.063 \\
\hline 1-propylnaphthalene & 1.428 & 1.105 & 0.000 & 0.297 & 7.068 \\
\hline 2-ethyl-7-methylnaphthalene & 1.460 & 0.962 & 0.000 & 0.293 & 7.079 \\
\hline 2-isopropylnaphthalene & 1.463 & 1.053 & 0.000 & 0.303 & 7.079 \\
\hline hexamethylbenzene & 0.969 & 0.711 & 0.000 & 0.318 & 7.092 \\
\hline 2-ethyl-3-methylnaphthalene & 1.488 & 1.027 & 0.000 & 0.301 & 7.094 \\
\hline 2,3,6-trimethylnaphthalene & 1.472 & 0.967 & 0.000 & 0.308 & 7.100 \\
\hline $\begin{array}{l}\text { (1E)-1-(2-propen-1-ylidene)-1,2,3,4- } \\
\text { tetrahydronaphthalene }\end{array}$ & 1.398 & 1.063 & 0.000 & 0.263 & 7.100 \\
\hline 4-[(1E)-1-propen-1-yl]-1,2-dihydronaphthalene & 1.399 & 1.077 & 0.000 & 0.267 & 7.109 \\
\hline 4-allyl-1,2-dihydronaphthalene & 1.390 & 1.067 & 0.000 & 0.263 & 7.111 \\
\hline 2-ethyl-6-methylnaphthalene & 1.456 & 0.972 & 0.000 & 0.295 & 7.113 \\
\hline 3-(2-methyl-propenyl)-1H-indene & 1.391 & 0.982 & 0.000 & 0.248 & 7.116 \\
\hline 1-(dimethylamino)naphthalene & 1.477 & 1.238 & 0.010 & 0.643 & 7.116 \\
\hline 2-allyl-3-methyl-1H-indene & 1.429 & 1.108 & 0.000 & 0.287 & 7.137 \\
\hline [(1E,3E)-6-methyl-1,3,5-heptatrien-1-yl]benzene & 0.983 & 0.919 & 0.000 & 0.267 & 7.142 \\
\hline 3-(3-buten-2-yl)-1H-indene & 1.446 & 1.067 & 0.010 & 0.264 & 7.142 \\
\hline 1-isopropylnaphthalene & 1.511 & 1.108 & 0.000 & 0.288 & 7.155 \\
\hline thalene & 1.497 & 1.027 & 0.000 & 0.291 & 7.186 \\
\hline 1,1'-(1,2-ethane & 1.348 & 1.056 & 0.000 & 0.342 & 7.187 \\
\hline en-1-yl)benzene & 0.925 & 0.884 & 0.000 & 0.292 & 7.191 \\
\hline 1,2,6-trimethylnap & 1.492 & 1.042 & 0.000 & 0.286 & 7.202 \\
\hline $1,2,7-\mathrm{t}$ & 1.493 & 1.042 & 0.000 & 0.280 & 7.222 \\
\hline 1,3,7-trimethyl & 1.468 & 1.056 & 0.000 & 0.282 & 7.222 \\
\hline 1-ethyl-8-methylnaphthalene & 1.524 & 1.092 & 0.000 & 0.275 & 7.225 \\
\hline 1,3,6-trimethylr & 1.473 & 1.072 & 0.000 & 0.275 & 7.237 \\
\hline nonylcyclopentene & 0.509 & 0.386 & 0.000 & 0.191 & 7.253 \\
\hline (3E)-1,3,7-octatrien-2-ylbenzene & 1.021 & 1.033 & 0.000 & 0.312 & 7.270 \\
\hline 1,2-dimethyl-3-hexylbenzene & 0.790 & 0.706 & 0.000 & 0.265 & 7.270 \\
\hline 1-benzyl-3-methylbenzene & 1.342 & 1.021 & 0.000 & 0.328 & 7.283 \\
\hline 1-benzyl-2-methylbenzene & 1.367 & 1.056 & 0.000 & 0.320 & 7.289 \\
\hline 1,1'-(1,1-ethanediyl)dibenzene & 1.409 & 1.100 & 0.000 & 0.322 & 7.293 \\
\hline 1-benzyl-4-methylbenzene & 1.324 & 1.048 & 0.000 & 0.331 & 7.302 \\
\hline hethylbiphenyl & 1.550 & 1.146 & 0.000 & 0.285 & 7.305 \\
\hline ethyl & 0.523 & 0.381 & 0.000 & 0.117 & 7.306 \\
\hline (2-allyl-1,4-pentadie & 1.024 & 1.047 & 0.000 & 0.310 & 7.311 \\
\hline 2,6-dimethylbiphe & 1.547 & 1.144 & 0.000 & 0.281 & 7.320 \\
\hline 1,4,6-trimethylnaphthalene & 1.482 & 1.103 & 0.000 & 0.253 & 7.325 \\
\hline pentylindan & 1.216 & 0.939 & 0.008 & 0.241 & 7.333 \\
\hline 1,4-pentadecadiene & 0.311 & 0.297 & 0.000 & 0.203 & 7.337 \\
\hline 2,4-dimethylbiphenyl & 1.512 & 1.186 & 0.000 & 0.302 & 7.337 \\
\hline
\end{tabular}




\begin{tabular}{|c|c|c|c|c|c|}
\hline 1-methyl-1-propylindan & 1.160 & 0.702 & 0.000 & 0.231 & 7.339 \\
\hline 1,3-pentadecadiene & 0.349 & 0.512 & 0.000 & 0.240 & 7.351 \\
\hline 1-[(3E)-1,3,6-heptatrien-4-yl]-2-methylbenzene & 1.113 & 1.069 & 0.000 & 0.298 & 7.351 \\
\hline 2,3'-dimethylbiphenyl & 1.523 & 1.158 & 0.000 & 0.295 & 7.352 \\
\hline 2,5-dimethylbiphenyl & 1.523 & 1.148 & 0.000 & 0.292 & 7.357 \\
\hline propylbutylcyclohexane & 0.551 & 0.379 & 0.000 & 0.113 & 7.362 \\
\hline 4-ethylbiphenyl & 1.401 & 1.192 & 0.000 & 0.331 & 7.363 \\
\hline 3,4-dimethylbiphenyl & 1.501 & 1.177 & 0.000 & 0.311 & 7.364 \\
\hline 1,2,3-trimethylnaphthalene & 1.501 & 1.108 & 0.000 & 0.264 & 7.371 \\
\hline 2,3-dimethylbiphenyl & 1.539 & 1.181 & 0.000 & 0.294 & 7.373 \\
\hline 1,2,5-trimethylnaphthalene & 1.502 & 1.108 & 0.000 & 0.264 & 7.376 \\
\hline 3-ethylbiphenyl & 1.436 & 1.213 & 0.000 & 0.325 & 7.383 \\
\hline 1,3,5-trimethylnaphthalene & 1.497 & 1.087 & 0.000 & 0.257 & 7.384 \\
\hline 1-methyl-2-heptylbenzene & 0.837 & 0.782 & 0.000 & 0.262 & 7.385 \\
\hline 2,4'-dimethylbiphenyl & 1.505 & 1.170 & 0.000 & 0.300 & 7.387 \\
\hline octylcyclohexane & 0.479 & 0.367 & 0.000 & 0.120 & 7.393 \\
\hline methylcyclohexylbenzene & 1.144 & 0.852 & 0.000 & 0.245 & 7.396 \\
\hline 3,5-dimethylbiphenyl & 1.506 & 1.164 & 0.000 & 0.302 & 7.402 \\
\hline 2-ethylbiphenyl & 1.484 & 1.199 & 0.000 & 0.319 & 7.403 \\
\hline 3,4,4a,9a-tetrahydrofluorene & 1.573 & 0.895 & 0.000 & 0.240 & 7.411 \\
\hline 3,3'-dimethylbiphenyl & 1.499 & 1.175 & 0.000 & 0.304 & 7.411 \\
\hline 1,3,8-trimethylnaphthalene & 1.501 & 1.102 & 0.000 & 0.244 & 7.419 \\
\hline 3,4'-dimethylbiphenyl & 1.470 & 1.146 & 0.000 & 0.311 & 7.424 \\
\hline 2-(3-buten-1-yl)naphthalene & 1.440 & 1.168 & 0.000 & 0.309 & 7.431 \\
\hline 2-methyl-1-methylene-2-vinylindane & 1.381 & 1.068 & 0.000 & 0.237 & 7.434 \\
\hline 4,4'-dimethylbiphenyl & 1.455 & 1.128 & 0.000 & 0.315 & 7.434 \\
\hline 1,2,4-trimethylnaphthalene & 1.533 & 1.121 & 0.000 & 0.253 & 7.455 \\
\hline pentadecene & 0.261 & 0.304 & 0.000 & 0.230 & 7.458 \\
\hline 1,2,8-trimethylnaphthalene & 1.528 & 1.120 & 0.000 & 0.255 & 7.459 \\
\hline propyltetralin & 1.264 & 0.981 & 0.000 & 0.238 & 7.482 \\
\hline 2-(2-methyl-2-propen-1-yl)naphthalene & 1.493 & 1.193 & 0.000 & 0.284 & 7.493 \\
\hline 1-tert-butyl-4-(2-methylprop-2-en-1-yl)benzene & 0.915 & 0.794 & 0.000 & 0.259 & 7.502 \\
\hline 1-(2-methyl-2-propen-1-yl)naphthalene & 1.554 & 1.203 & 0.000 & 0.274 & 7.514 \\
\hline isopentadecane & 0.201 & 0.170 & 0.000 & 0.117 & 7.515 \\
\hline 1,4,5-trimethylnaphthalene & 1.529 & 1.113 & 0.000 & 0.235 & 7.552 \\
\hline propyldecalin & 1.047 & 0.607 & 0.000 & 0.131 & 7.563 \\
\hline octylcyclohexene & 0.534 & 0.391 & 0.000 & 0.183 & 7.570 \\
\hline 1-allyl-4-methylene-1,4-dihydronaphthalene & 1.571 & 1.194 & 0.000 & 0.266 & 7.571 \\
\hline 1-ethyl-2-hexylbenzene & 0.867 & 0.736 & 0.000 & 0.250 & 7.576 \\
\hline$n$-pentadecane & 0.204 & 0.169 & 0.000 & 0.094 & 7.591 \\
\hline hexadecyne & 0.204 & 0.169 & 0.000 & 0.094 & 7.591 \\
\hline 1-propyl-2-pentylbenzene & 0.897 & 0.762 & 0.000 & 0.247 & 7.620 \\
\hline 1-butyl-2-butylbenzene & 0.901 & 0.763 & 0.000 & 0.245 & 7.634 \\
\hline
\end{tabular}




\begin{tabular}{|c|c|c|c|c|c|}
\hline (3-cyclopentylpropyl)benzene & 0.998 & 0.855 & 0.000 & 0.251 & 7.640 \\
\hline [(E)-2-(1-cyclohexen-1-yl)vinyl]benzene & 1.236 & 1.128 & 0.000 & 0.313 & 7.665 \\
\hline phenethylcyclohexane & 1.073 & 0.877 & 0.000 & 0.247 & 7.725 \\
\hline cyclotetradecene & 0.585 & 0.355 & 0.000 & 0.168 & 7.743 \\
\hline 1,1,2-trimethyl-1,2-dihydronaphthalene & 1.358 & 1.019 & 0.000 & 0.235 & 7.747 \\
\hline 2-butylnaphthalene & 1.375 & 1.226 & 0.000 & 0.313 & 7.801 \\
\hline methylheptylcyclohexane & 0.540 & 0.419 & 0.000 & 0.124 & 7.809 \\
\hline decylcyclopentene & 0.526 & 0.432 & 0.000 & 0.189 & 7.811 \\
\hline 1,1,6-trimethyl-1,2,3,4-tetrahydronaphthalene & 1.282 & 0.816 & 0.000 & 0.202 & 7.815 \\
\hline 1,2,4,5-tetraethylbenzene & 1.083 & 0.819 & 0.000 & 0.235 & 7.818 \\
\hline 2-sec-butylnaphthalene & 1.500 & 1.238 & 0.000 & 0.307 & 7.818 \\
\hline 2-isobutylnaphthalene & 1.451 & 1.189 & 0.000 & 0.304 & 7.822 \\
\hline (4-vinyl-3-cyclohexen-1-yl)benzene & 1.221 & 0.941 & 0.000 & 0.261 & 7.831 \\
\hline 1-butylnaphthalene & 1.441 & 1.239 & 0.000 & 0.308 & 7.834 \\
\hline 1,2,3,4-tetraethylbenzene & 1.109 & 0.822 & 0.000 & 0.232 & 7.838 \\
\hline cyclohexylethylbenzene & 1.208 & 0.911 & 0.000 & 0.244 & 7.839 \\
\hline 1,1,5,6-tetramethylindane & 1.354 & 0.774 & 0.000 & 0.222 & 7.840 \\
\hline hexylcyclooctene & 0.545 & 0.333 & 0.000 & 0.185 & 7.841 \\
\hline 1,2-dimethyl-3-heptylbenzene & 0.797 & 0.729 & 0.000 & 0.265 & 7.843 \\
\hline heptylketone & 0.296 & 0.803 & 0.000 & 0.568 & 7.844 \\
\hline cyclotetradecane & 0.577 & 0.291 & 0.000 & 0.103 & 7.847 \\
\hline hexylindan & 1.280 & 0.994 & 0.000 & 0.241 & 7.859 \\
\hline (4,5-dimethyl-1,4-cyclohexadien-1-yl)benzene & 1.415 & 1.064 & 0.000 & 0.239 & 7.861 \\
\hline 1-(1,5-cyclohexadien-1-yl)-3,5-dimethylbenzene & 1.409 & 1.117 & 0.000 & 0.260 & 7.868 \\
\hline 1-isobutylnaphthalene & 1.509 & 1.197 & 0.000 & 0.288 & 7.873 \\
\hline 4-cyclohexyl-1,2-dimethylbenzene & 1.293 & 0.914 & 0.000 & 0.237 & 7.873 \\
\hline 1,6,8-trimethyl-1,2,3,4-tetrahydronaphthalene & 1.391 & 0.866 & 0.000 & 0.204 & 7.880 \\
\hline 1,1,4-trimethyl-1,2,3,4-tetrahydronaphthalene & 1.348 & 0.823 & 0.000 & 0.204 & 7.882 \\
\hline butyltetralin & 1.320 & 1.113 & 0.000 & 0.239 & 7.890 \\
\hline 2,6-diethylnaphthalene & 1.476 & 1.131 & 0.000 & 0.281 & 7.895 \\
\hline 6-butyltetralin & 1.218 & 1.003 & 0.000 & 0.246 & 7.897 \\
\hline 2-methyl-1-propylnaphthalene & 1.565 & 1.178 & 0.000 & 0.268 & 7.899 \\
\hline 1-methyl-4-propylnaphthalene & 1.568 & 1.218 & 0.000 & 0.280 & 7.900 \\
\hline 1-methyl-4-(4-methylcyclohexyl)benzene & 1.252 & 0.913 & 0.000 & 0.217 & 7.903 \\
\hline 2-allyl-3-methyl-1,2-dihydronaphthalene & 1.506 & 1.191 & 0.000 & 0.277 & 7.906 \\
\hline dibenzylmethane & 1.279 & 1.144 & 0.000 & 0.335 & 7.912 \\
\hline 4-[(2S)-3-buten-2-yl]-1,2-dihydronaphthalene & 1.525 & 1.182 & 0.000 & 0.249 & 7.914 \\
\hline 1-sec-butylnaphthalene & 1.552 & 1.255 & 0.000 & 0.288 & 7.915 \\
\hline 1,2-diethylnaphthalene & 1.581 & 1.116 & 0.000 & 0.254 & 7.925 \\
\hline 1-isobutyl-1,2,3,4-tetrahydronaphthalene & 1.406 & 1.056 & 0.000 & 0.231 & 7.929 \\
\hline dimethylcyclohexylbenzene & 1.373 & 0.907 & 0.000 & 0.228 & 7.937 \\
\hline 5-isobutyl-1,2,3,4-tetrahydronaphthalene & 1.385 & 0.969 & 0.000 & 0.235 & 7.939 \\
\hline 2-isopropyl-6-methylnaphthalene & 1.515 & 1.123 & 0.000 & 0.289 & 7.942 \\
\hline
\end{tabular}




\begin{tabular}{|c|c|c|c|c|c|}
\hline 1-methyl-3-(2-phenylethyl)benzene & 1.286 & 1.125 & 0.000 & 0.324 & 7.948 \\
\hline 1,3-diethylnaphthalene & 1.565 & 1.223 & 0.000 & 0.276 & 7.957 \\
\hline nonylbenzene & 0.848 & 0.911 & 0.000 & 0.251 & 7.960 \\
\hline tetrahydroanthracene & 1.758 & 1.282 & 0.000 & 0.448 & 7.961 \\
\hline 1-methyl-2-(2-phenylethyl)benzene & 1.315 & 1.174 & 0.000 & 0.322 & 7.965 \\
\hline 2-tert-butylnaphthalene & 1.503 & 1.176 & 0.000 & 0.267 & 7.966 \\
\hline 1-methyl-4-(2-phenylethyl)benzene & 1.277 & 1.138 & 0.000 & 0.325 & 7.979 \\
\hline 1-methyl-7-isopropylnaphthalene & 1.554 & 1.097 & 0.000 & 0.266 & 8.004 \\
\hline $\begin{array}{l}\text { (2,3-dimethyl-5-methylene-2-cyclopenten-1- } \\
\text { yl)benzene }\end{array}$ & 1.520 & 0.910 & 0.000 & 0.211 & 8.012 \\
\hline 1-isopropyl-8-methylnaphthalene & 1.590 & 1.095 & 0.000 & 0.249 & 8.016 \\
\hline 1-isopropyl-4-me & 1.584 & 1.093 & 0.000 & 0.246 & 8.024 \\
\hline 1-benzyl-2-ethylbenzene & 1.408 & 1.193 & 0.000 & 0.318 & 8.028 \\
\hline 1,1'-(1,2-prop & 1.373 & 1.228 & 0.000 & 0.333 & 8.036 \\
\hline 6-tert-butyltetralin & 1.334 & 0.916 & 0.000 & 0.228 & 8.044 \\
\hline 1,1-dif & 1.480 & 1.253 & 0.000 & 0.328 & 8.050 \\
\hline tetrah & 1.764 & 1.278 & 0.000 & 0.438 & 8.051 \\
\hline $1-\mathrm{met}$ & 0.891 & 0.860 & 0.000 & 0.252 & 8.065 \\
\hline butyld & 1.170 & 0.674 & 0.000 & 0.141 & 8.066 \\
\hline -ethylbenzene & 1.318 & 1.188 & 0.000 & 0.324 & 8.074 \\
\hline 1 -tert- & 1.520 & 1.181 & 0.000 & 0.250 & 8.075 \\
\hline 1-ethy & 0.924 & 0.843 & 0.000 & 0.235 & 8.080 \\
\hline 1-benzyl-3, & 1.441 & 1.076 & 0.000 & 0.302 & 8.082 \\
\hline 1-methyl-1-bu & 1.226 & 0.696 & 0.000 & 0.231 & 8.088 \\
\hline 3-(3-buten-2-yl)-2-methyl-1H-indene & 1.552 & 1.064 & 0.000 & 0.215 & 8.090 \\
\hline hexadecene & 0.276 & 0.312 & 0.000 & 0.216 & 8.093 \\
\hline -4-ethylbenzene & 1.300 & 1.176 & 0.000 & 0.325 & 8.093 \\
\hline 2-prop & 1.486 & 1.323 & 0.000 & 0.323 & 8.095 \\
\hline 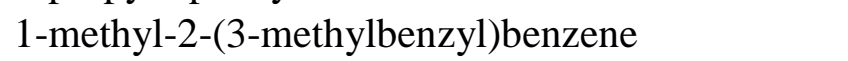 & 1.483 & 1.109 & 0.000 & 0.299 & 8.098 \\
\hline 4-benzyl-1,2-dimethyl & 1.462 & 1.174 & 0.000 & 0.312 & 8.102 \\
\hline 2,5-dimethyldiphenyln & 1.498 & 1.108 & 0.000 & 0.297 & 8.104 \\
\hline 1,1'-methylenebis(3-methylbenzene) & 1.407 & 1.093 & 0.000 & 0.305 & 8.108 \\
\hline (4,5,5-trimethyl-1,3-cyclopentadien-1-yl)benzene & 1.393 & 1.184 & 0.000 & 0.234 & 8.109 \\
\hline 2,2',6-trimethylbiphenyl & 1.657 & 1.184 & 0.000 & 0.255 & 8.117 \\
\hline 1-methyl-2-(1-phenylethyl)benzene & 1.535 & 1.177 & 0.000 & 0.308 & 8.124 \\
\hline $1,1^{\prime}$-methylenebis(2-methylb & 1.508 & 1.115 & 0.000 & 0.296 & 8.127 \\
\hline 1-methyl-2-(4-methyl & 1.451 & 1.104 & 0.000 & 0.303 & 8.127 \\
\hline 1-met & 1.398 & 1.111 & 0.000 & 0.310 & 8.128 \\
\hline 1-ben & 1.493 & 1.172 & 0.000 & 0.304 & 8.129 \\
\hline pylbiphenyl & 1.563 & 1.271 & 0.000 & 0.308 & 8.130 \\
\hline & 0.217 & 0.176 & 0.000 & 0.105 & 8.132 \\
\hline 2-benzyl-1,3 & 1.516 & 1.120 & 0.000 & 0.289 & 8.132 \\
\hline 1,1'-(2,2-propanediyl)dibenzene & 1.421 & 1.289 & 0.000 & 0.292 & 8.138 \\
\hline
\end{tabular}




\begin{tabular}{|c|c|c|c|c|c|}
\hline 1-propyl-2-hexylbenzene & 0.962 & 0.865 & 0.000 & 0.234 & 8.142 \\
\hline 5,8-dimethyl-3-vinyl-1,2-dihydronaphthalene & 1.552 & 1.124 & 0.000 & 0.214 & 8.147 \\
\hline 2-(2-methyl-3-buten-2-yl)-1H-indene & 1.432 & 1.127 & 0.000 & 0.227 & 8.151 \\
\hline 3-propylbiphenyl & 1.434 & 1.321 & 0.000 & 0.330 & 8.152 \\
\hline 1-ethyl-1,2-dihydroacenaphthylene & 1.740 & 1.218 & 0.000 & 0.356 & 8.153 \\
\hline 2,4,6-trimethylbiphenyl & 1.642 & 1.217 & 0.000 & 0.261 & 8.156 \\
\hline 1-butyl-2-pentylbenzene & 0.972 & 0.866 & 0.000 & 0.230 & 8.161 \\
\hline 4-propylbiphenyl & 1.396 & 1.341 & 0.000 & 0.333 & 8.164 \\
\hline cyclopentadecene & 0.590 & 0.367 & 0.000 & 0.163 & 8.172 \\
\hline$n$-hexadecane & 0.217 & 0.175 & 0.000 & 0.092 & 8.174 \\
\hline heptadecyne & 0.217 & 0.175 & 0.000 & 0.092 & 8.174 \\
\hline 3-isopropylbiphenyl & 1.486 & 1.301 & 0.000 & 0.322 & 8.174 \\
\hline 1,1-diethyl-1,2,3,4-tetrahydronaphthalene & 1.347 & 0.933 & 0.000 & 0.185 & 8.179 \\
\hline 1-methyl-4-(1-phenylethyl)benzene & 1.493 & 1.103 & 0.000 & 0.313 & 8.179 \\
\hline 1,2,4-tripropylbenzene & 1.047 & 0.918 & 0.000 & 0.240 & 8.190 \\
\hline 2-phenyl-1,2,3,4,5,6-hexahydropentalene & 1.515 & 0.994 & 0.000 & 0.243 & 8.195 \\
\hline 1,1'-methylenebis(4-methylbenzene) & 1.371 & 1.109 & 0.000 & 0.311 & 8.196 \\
\hline 2,3',5-trimethylbiph & 1.604 & 1.230 & 0.000 & 0.277 & 8.198 \\
\hline 4-isopropylbiphenyl & 1.470 & 1.318 & 0.000 & 0.325 & 8.199 \\
\hline 1-(4-penten-1-yl)naphthalene & 1.497 & 1.281 & 0.000 & 0.295 & 8.204 \\
\hline [5-(3-buten-1-yl)-1,4-cyc & 1.470 & 1.245 & 0.000 & 0.271 & 8.212 \\
\hline 1-methyl-4,9-dihydro-1H-fluorene & 1.742 & 1.179 & 0.000 & 0.290 & 8.220 \\
\hline nonylcyclohexene & 0.554 & 0.459 & 0.000 & 0.188 & 8.230 \\
\hline ten-1-yl)naphthalene & 1.457 & 1.254 & 0.000 & 0.284 & 8.230 \\
\hline $1,3,6,8$-tetramethy & 1.592 & 1.142 & 0.000 & 0.225 & 8.231 \\
\hline $\begin{array}{l}\text { 6-methoxy-1,1-dimethyl-1,2,3,4- } \\
\text { tetrahydronaphthalene }\end{array}$ & 1.368 & 1.141 & 0.010 & 0.546 & 8.231 \\
\hline 2,4',5-trimethylbiphenyl & 1.599 & 1.251 & 0.000 & 0.284 & 8.234 \\
\hline 3,4',5-trimethylbi & 1.576 & 1.268 & 0.000 & 0.289 & 8.245 \\
\hline 2,2,5,7-tetra & 1.460 & 0.898 & 0.000 & 0.214 & 8.264 \\
\hline 1,3,5,7-tetramethylnaphthale & 1.598 & 1.152 & 0.000 & 0.230 & 8.270 \\
\hline 1-[(2E)-2-penten-3-yl]naphthalene & 1.643 & 1.279 & 0.000 & 0.279 & 8.277 \\
\hline 2,3,6,7-tetramethylnaphthalene & 1.593 & 1.160 & 0.000 & 0.248 & 8.283 \\
\hline cyclopentadecane & 0.594 & 0.306 & 0.000 & 0.105 & 8.287 \\
\hline nonylcyclohexane & 0.516 & 0.443 & 0.000 & 0.118 & 8.295 \\
\hline 1-cyclopentyl-1H-indene & 1.617 & 1.136 & 0.010 & 0.288 & 8.295 \\
\hline 2,4,4'-trimethylbiphenyl & 1.588 & 1.263 & 0.000 & 0.287 & 8.302 \\
\hline 4-ethyl-4'-methylb & 1.483 & 1.260 & 0.000 & 0.314 & 8.303 \\
\hline 1-(1-penten-2-yl)na & 1.621 & 1.317 & 0.000 & 0.289 & 8.307 \\
\hline 2-(4-penten-2-yl)naphthalene & 1.558 & 1.310 & 0.000 & 0.336 & 8.314 \\
\hline 1,2,4,7-tetramethylnaphthalene & 1.599 & 1.154 & 0.000 & 0.227 & 8.316 \\
\hline 1,2,3,4,5,6-hexahydroanthracene & 1.666 & 1.190 & 0.000 & 0.414 & 8.323 \\
\hline 1,4,5,8-tetramethylnaphthalene & 1.624 & 1.148 & 0.000 & 0.224 & 8.324 \\
\hline
\end{tabular}




\begin{tabular}{|c|c|c|c|c|c|}
\hline 1,2,6,7-tetramethylnaphthalene & 1.596 & 1.163 & 0.000 & 0.243 & 8.329 \\
\hline 1,3,6,7-tetramethylnaphthalene & 1.591 & 1.172 & 0.000 & 0.234 & 8.344 \\
\hline octylcycloheptane & 0.439 & 0.279 & 0.000 & 0.112 & 8.346 \\
\hline 1,2,3,4-tetramethylnaphthalene & 1.624 & 1.143 & 0.000 & 0.242 & 8.348 \\
\hline 1,2,3,7-tetramethylnaphthalene & 1.601 & 1.164 & 0.000 & 0.244 & 8.353 \\
\hline 1,2,5,7-tetramethylnaphthalene & 1.601 & 1.162 & 0.000 & 0.224 & 8.359 \\
\hline undecylcyclopentene & 0.542 & 0.478 & 0.000 & 0.187 & 8.368 \\
\hline 1,4,6,7-tetramethylnaphthalene & 1.599 & 1.174 & 0.000 & 0.230 & 8.368 \\
\hline 1,1,3,3,5-pentamethylindane & 1.439 & 0.839 & 0.000 & 0.210 & 8.376 \\
\hline 2-ethyl-7-methoxy-1-methylnaphthalene & 1.609 & 1.352 & 0.010 & 0.553 & 8.378 \\
\hline heptylindan & 1.345 & 1.049 & 0.000 & 0.241 & 8.385 \\
\hline 1,2,3,6-tetramethylnaphthalene & 1.589 & 1.165 & 0.000 & 0.238 & 8.386 \\
\hline 1,2,5,6-tetramethylnaphthalene & 1.595 & 1.144 & 0.000 & 0.244 & 8.403 \\
\hline 4a,8a,9,9a,10,10a-hexahydroanthracene & 1.641 & 0.928 & 0.000 & 0.391 & 8.413 \\
\hline 1,2-dimethyl-3-octylbenzene & 0.805 & 0.752 & 0.000 & 0.265 & 8.416 \\
\hline 1,1,4,4-tetramethyl-1,2,3,4-tetrahydronaphthalene & 1.407 & 0.884 & 0.000 & 0.182 & 8.418 \\
\hline decylbenzene & 0.918 & 0.978 & 0.000 & 0.235 & 8.420 \\
\hline 1,2,3,4,4a,10a-hexahydrophenanthrene & 1.709 & 1.187 & 0.000 & 0.408 & 8.433 \\
\hline $1,4,5,8,9,10$-hexahydroanthracene & 1.584 & 0.830 & 0.000 & 0.326 & 8.440 \\
\hline $1,2,3,9,10,10$ a-hexahydrophenanthrene & 1.678 & 1.175 & 0.000 & 0.379 & 8.440 \\
\hline $1,2,3,4,9,10$-hexahydrophenanthrene & 1.673 & 1.214 & 0.000 & 0.369 & 8.441 \\
\hline isopropylcyclohexylbenzene & 1.415 & 1.014 & 0.000 & 0.260 & 8.472 \\
\hline 1,1,2,3,3-pentamethylindane & 1.522 & 0.852 & 0.000 & 0.215 & 8.478 \\
\hline 7-sec-butyl-1-methylnaphthalene & 1.611 & 1.271 & 0.000 & 0.272 & 8.486 \\
\hline 1-methyl-2-nonylbenzene & 0.959 & 0.944 & 0.000 & 0.239 & 8.496 \\
\hline butylatedhydroxytoluene & 1.210 & 1.135 & 0.420 & 0.613 & 8.530 \\
\hline pentaethyl & 1.268 & 0.945 & 0.000 & 0.227 & 8.567 \\
\hline isoheptadec & 0.224 & 0.195 & 0.000 & 0.119 & 8.598 \\
\hline tylbenzene & 1.027 & 0.990 & 0.000 & 0.220 & 8.624 \\
\hline $\begin{array}{l}\text { 8-isopropyl-2,5-dimethyl-1,2,3,4- } \\
\text { tetrahydronaphthalene }\end{array}$ & 1.568 & 0.981 & 0.000 & 0.213 & 8.633 \\
\hline $\begin{array}{l}\text { (1S,4S)-4-isopropyl-1,6-dimethyl-1,2,3,4- } \\
\text { tetrahydronaphthalene }\end{array}$ & 1.586 & 0.985 & 0.000 & 0.215 & 8.634 \\
\hline heptadecene & 0.284 & 0.334 & 0.000 & 0.230 & 8.635 \\
\hline $\begin{array}{l}\text { 6-isopropyl-1,1-dimethyl-1,2,3,4- } \\
\text { tetrahydronaphthalene }\end{array}$ & 1.484 & 0.964 & 0.000 & 0.227 & 8.647 \\
\hline 1-ethyl-2-octylbenzene & 0.979 & 0.930 & 0.000 & 0.223 & 8.652 \\
\hline decylcyclohexane & 0.551 & 0.463 & 0.000 & 0.124 & 8.663 \\
\hline 1-propyl-2-heptylbenzene & 1.008 & 0.960 & 0.000 & 0.222 & 8.663 \\
\hline 1-butyl-2-hexylbenzene & 1.021 & 0.981 & 0.000 & 0.221 & 8.667 \\
\hline pentyltetralin & 1.447 & 1.201 & 0.000 & 0.242 & 8.685 \\
\hline $\begin{array}{l}\text { 7-ethyl-1,1,4-trimethyl-1,2,3,4- } \\
\text { tetrahydronaphthalene }\end{array}$ & 1.563 & 0.981 & 0.000 & 0.219 & 8.709 \\
\hline
\end{tabular}




\begin{tabular}{|c|c|c|c|c|c|}
\hline 1-(2,2-dimethylcyclopropyl)naphthalene & 1.631 & 1.142 & 0.000 & 0.209 & 8.712 \\
\hline 2-methyl-1,2,3,4-tetrahydrophenanthrene & 1.800 & 1.237 & 0.000 & 0.292 & 8.723 \\
\hline cyclohexadecene & 0.624 & 0.378 & 0.000 & 0.168 & 8.749 \\
\hline$n$-heptadecane & 0.223 & 0.203 & 0.000 & 0.109 & 8.760 \\
\hline octadecyne & 0.223 & 0.203 & 0.000 & 0.109 & 8.760 \\
\hline 1,2-dipropylnaphthalene & 1.696 & 1.302 & 0.000 & 0.287 & 8.810 \\
\hline 1-methyl-1-pentylindan & 1.292 & 0.689 & 0.000 & 0.231 & 8.838 \\
\hline nonylcycloheptane & 0.469 & 0.276 & 0.000 & 0.117 & 8.845 \\
\hline 2,6-diisopropylnaphthalene & 1.669 & 1.346 & 0.000 & 0.272 & 8.898 \\
\hline cyclohexadecane & 0.606 & 0.319 & 0.000 & 0.106 & 8.903 \\
\hline octylindan & 1.409 & 1.104 & 0.000 & 0.241 & 8.912 \\
\hline 1,2-dimethyl-3-nonylbenzene & 0.812 & 0.775 & 0.000 & 0.265 & 8.989 \\
\hline dodecylcyclopentene & 0.565 & 0.529 & 0.000 & 0.185 & 9.054 \\
\hline undecylbenzene & 0.937 & 1.043 & 0.000 & 0.226 & 9.098 \\
\hline 1-methyl-2-decylbenzene & 0.933 & 0.939 & 0.000 & 0.249 & 9.104 \\
\hline octylketone & 0.319 & 0.866 & 0.000 & 0.599 & 9.156 \\
\hline isooctadecane & 0.230 & 0.224 & 0.000 & 0.116 & 9.291 \\
\hline Undecylcyclohexane- & 0.589 & 0.502 & 0.000 & 0.127 & 9.318 \\
\hline 1-butyl-2-heptylbenzene & 1.094 & 1.040 & 0.000 & 0.240 & 9.321 \\
\hline 1-propyl-2-octylbenzene & 1.072 & 1.019 & 0.000 & 0.240 & 9.340 \\
\hline 1-penty & 1.111 & 1.047 & 0.000 & 0.240 & 9.348 \\
\hline 1-ethyl-2-nonylbenzene & 1.001 & 0.954 & 0.000 & 0.242 & 9.348 \\
\hline octadecene & 0.286 & 0.357 & 0.000 & 0.238 & 9.370 \\
\hline nonylindan & 1.474 & 1.159 & 0.000 & 0.241 & 9.438 \\
\hline$n$-octadecane & 0.226 & 0.235 & 0.000 & 0.111 & 9.445 \\
\hline cyclohe & 0.605 & 0.342 & 0.000 & 0.113 & 9.533 \\
\hline 3-decylbenzene & 0.820 & 0.798 & 0.000 & 0.265 & 9.563 \\
\hline 1-methyl-1-hexylindan & 1.358 & 0.683 & 0.000 & 0.231 & 9.587 \\
\hline dodec & 0.950 & 1.093 & 0.000 & 0.223 & 9.670 \\
\hline yl-2-undecylbenzene & 0.960 & 0.984 & 0.000 & 0.249 & 9.694 \\
\hline tridecylcyclopentene & 0.588 & 0.580 & 0.000 & 0.184 & 9.711 \\
\hline 1,2-dihexylbenzene & 1.057 & 1.135 & 0.000 & 0.208 & 9.822 \\
\hline dodecylcyclohexane & 0.635 & 0.573 & 0.000 & 0.132 & 9.829 \\
\hline hexaethylbenzene & 1.520 & 1.098 & 0.000 & 0.252 & 9.868 \\
\hline $\begin{array}{l}\text { 1,4-diethyl-1,4,6-trimethyl-1,2,3,4- } \\
\text { tetrahydronaphthalene }\end{array}$ & 1.553 & 1.133 & 0.000 & 0.223 & 9.933 \\
\hline 1-propyl-2-nonylbenzene & 1.131 & 1.095 & 0.000 & 0.240 & 9.952 \\
\hline 1-pentyl-2-heptylbenzene & 1.180 & 1.129 & 0.000 & 0.240 & 9.960 \\
\hline 1-hexyl-2-hexylbenzene & 1.192 & 1.134 & 0.000 & 0.240 & 9.970 \\
\hline 1-ethyl-2-decylbenzene & 1.044 & 1.012 & 0.000 & 0.242 & 9.976 \\
\hline 1,2,4-tributylbenzene & 1.173 & 1.052 & 0.000 & 0.255 & 10.066 \\
\hline tetrapropylbenzene & 1.177 & 1.033 & 0.000 & 0.247 & 10.079 \\
\hline 1,1-di-tert-butyl-1,2,3,4-tetrahydronaphthalene & 1.518 & 1.096 & 0.000 & 0.226 & 10.092 \\
\hline
\end{tabular}




\begin{tabular}{llllll}
\hline cyclooctadecane & 0.607 & 0.360 & 0.000 & 0.116 & 10.130 \\
1,2-dimethyl-3-undecylbenzene & 0.827 & 0.821 & 0.000 & 0.265 & 10.136 \\
1,3-dimethyl-2-phenylnaphthalene & 2.260 & 1.603 & 0.000 & 0.386 & 10.248 \\
$\begin{array}{l}\text { 6,7-diethyl-1,1,4,4-tetramethyl-1,2,3,4- } \\
\text { tetrahydronaphthalene }\end{array}$ & 1.580 & 1.247 & 0.000 & 0.286 & 10.254 \\
\hline
\end{tabular}




\section{Abbreviation List}

$\alpha$, separation factor

$\gamma$, heating rate

5ms, 5\% (phenyl)methylpolysiloxane

$a$, stationary phase contributions to the interactions of hydrogen bond with acid functionalities

$A$, analyte contributions to the interactions of hydrogen bond with acid functionalities

$A_{o}$, scale which is calculated according to the peak distribution in the normalized chromatogram relative to four main asterisked axes in the $2 \mathrm{D}$ space by ignoring the bin coverage

analytes $_{\text {total }}$, total number of separated analytes

$b$, stationary phase contributions to the interactions of hydrogen bond with basic functionalities $B$, analyte contributions to the interactions of hydrogen bond with basic functionalities

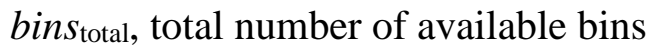

sbins, number of bins containing peaks

$c$, an intercept constant (LSER)

$C_{\text {peak }}$, correlation of distribution pattern of $2 \mathrm{D}$ peaks

$C_{\text {pert }}$, bin coverage ratio

${ }^{1} \mathrm{D}$, first dimension

${ }^{2} \mathrm{D}$, second dimension

1D, one dimensional

1DGC, one dimensional gas chromatography

2D, two dimensional

2DGC, two dimensional gas chromatography

3D, three dimensional

DS, Deans switch

$e$, stationary phase contributions to the interactions of dispersity

$E$, analyte contributions to the interactions of dispersity

${ }^{1} F$, flow rate in the first dimension

${ }^{2} F$, flow rate in the second dimension

GC, gas chromatography

$\mathrm{GC} \times \mathrm{GC}$, comprehensive two dimensional gas chromatography

$H$, plate height 
$\mathrm{H} / \mathrm{C}$, heart-cut

IL, ionic liquid

${ }^{i} k_{j}$, analyte retention for analyte $j$ in column $i$

$l$, stationary phase contributions to the interactions of dispersion/cavity formation for gas to liquid phase

$L_{L S E R}$, analyte contributions to the interactions of dispersion/cavity formation for gas to liquid phase

$L$, column length

${ }^{1} L$, first dimension column length

${ }^{2} L$, second dimension column length

LSER, linear solvation energy relationship

${ }^{i} m$, a whole number representing the number of separation dimensions

MDGC, multidimensional gas chromatography;

$M_{\mathrm{R}}$, modulation ratio (the ratio of the ${ }^{1} \mathrm{D}$ peak width to modulation period)

$n_{\mathrm{c}}$, column peak capacity

${ }^{1} n_{\mathrm{c}}$, column peak capacity in first dimension

${ }^{2} n_{\mathrm{c}}$, column peak capacity in second dimension

$N_{i n j}$, number of injections

$N_{\text {plate, }}$ number of plates

$O$, orthogonality

$P_{a p p}$, apparent number of separated peaks

${ }^{1} P_{\text {app }}$, apparent number of separated peaks in ${ }^{1} \mathrm{D}$ separation

${ }^{2} P_{\text {app }}$, apparent number of peaks in ${ }^{2} \mathrm{D}$ separation

$P_{\text {app,total }}$, total apparent number of peaks that a system can separate

$P_{\mathrm{M}}$, modulation period

$s$, stationary phase contributions to the interactions of dipolarity

$S$, analyte contributions to the interactions of dipolarity

$t_{\text {cyc }}$, cycle time

$t_{\mathrm{R}}$, retention time

${ }^{1} t_{\mathrm{R}}$, first dimension retention time

${ }^{2} t_{\mathrm{R}}$, second dimension retention time

${ }^{i} t_{\mathrm{R}, \mathrm{j}}$, time when the analyte $j$ elutes from the column $i$ 
${ }^{i} t_{0, j}$, time when the analyte $j$ enters the column $i$

${ }^{i} t_{M}(T)$, the column $i$ unretained solute time as a function of temperature

$t_{\mathrm{R}, \max }$, retention time of the most retained analyte

${ }^{i} t_{\mathrm{R}, \max }$, retention time of the most retained analyte in column $i$

${ }^{2} t_{\mathrm{R}, \max }$, last eluting analyte on the second dimension column

$t_{\mathrm{R}, \mathrm{min}}$, retention time of the least retained analyte

$i_{t_{\mathrm{R}, \min }}$, retention time of the least retained analyte in column $i$

$t_{\mathrm{R}, \text { normalized, }}$, normalized retention time

${ }^{1} t_{\mathrm{R}, \text { normalized }}$, first dimension normalized retention time

${ }^{2} t_{\mathrm{R}, \text { normalized }}$, second dimension normalized retention time

$\Delta t_{\mathrm{R}}$, difference in retention times of any two adjacent peaks

$\Delta^{1} t_{\mathrm{R}}$, difference in retention times of any two adjacent peaks in ${ }^{1} \mathrm{D}$ separation

$\Delta^{2} t_{R}$, difference in retention times of any two adjacent peaks in ${ }^{2} \mathrm{D}$ separation

$t_{\text {samp }}$, sampling time

$T$, temperature

$T_{R}$, elution temperature

${ }^{i} T_{R, j}$, elution temperature for analyte $j$ in column $i$

${ }^{i} T_{0, j}$, temperature at the time when the analyte $j$ enters the column $i$

$w_{b}$, average peak width at baseline

${ }^{1} w_{b}$, average peak width at baseline in the first dimension

${ }^{2} w_{b}$, average peak width at baseline in the second dimension

$w_{\mathrm{h}}$, peak width at half height

WAX, polyethyleneglycol

\section{References}

(1) Mitrevski, B.; Webster, R. L.; Rawson, P.; Evans, D. J.; Choi, H. K.; Marriott, P. J. J. Chromatogr. A 2012, 1224, 89-96.

(2) Webster, R. L.; Evans, D. J.; Marriott, P. J. Energy \& Fuels 2015, 29, 2059-2066. 